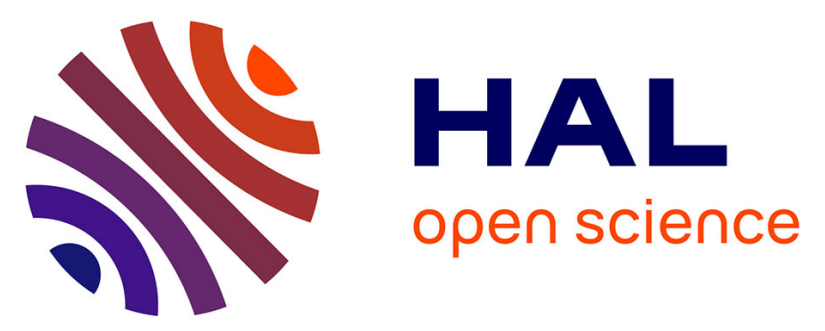

\title{
MODAL ANALYSIS OF MECHANICAL SYSTEMS WITH IMPACT NON-LINEARITIES: LIMITATIONS TO A MODAL SUPERPOSITION
}

Claude-Henri Lamarque, Olivier Janin

\section{- To cite this version:}

Claude-Henri Lamarque, Olivier Janin. MODAL ANALYSIS OF MECHANICAL SYSTEMS WITH IMPACT NON-LINEARITIES: LIMITATIONS TO A MODAL SUPERPOSITION. Journal of Sound and Vibration, 2000, 235, pp.567 - 609. 10.1006/jsvi.1999.2932 . hal-01510826

\section{HAL Id: hal-01510826 \\ https://hal.science/hal-01510826}

Submitted on 20 Apr 2017

HAL is a multi-disciplinary open access archive for the deposit and dissemination of scientific research documents, whether they are published or not. The documents may come from teaching and research institutions in France or abroad, or from public or private research centers.
L'archive ouverte pluridisciplinaire HAL, est destinée au dépôt et à la diffusion de documents scientifiques de niveau recherche, publiés ou non, émanant des établissements d'enseignement et de recherche français ou étrangers, des laboratoires publics ou privés.

\section{(c)(1)}

Distributed under a Creative Commons Attribution| 4.0 International License 


\title{
MODAL ANALYSIS OF MECHANICAL SYSTEMS WITH IMPACT NON-LINEARITIES: LIMITATIONS TO A MODAL SUPERPOSITION
}

\author{
C. H. Lamarque and O. Janin \\ Ecole Nationale des Travaux Publics de l'Etat, Laboratoire GéoMatériaux, URA-CNRS 1652, \\ Rue Maurice Audin, 69518 Vaulx-En-Velin Cedex, France
}

\begin{abstract}
This paper presents an attempt to generalize the modal superposition formula to mechanical systems with impact type non-linearities following the procedure introduced for smooth non-linearities. The study is restricted to simple one- and two-degree-of-freedom systems with a unilateral constraint on one of the degrees of freedom, for which the response can be analytically determined. Generalized frequencies, modes and masses are built in the procedure. The results obtained for various sets of parameters point out some limitations to the validity of a general modal superposition formula.
\end{abstract}

\section{INTRODUCTION}

Analysis of the response of structures is convenient if a linear model can fully describe the structure. Within this framework, it is useful to introduce in either the finite or infinite dimension (Hilbertian case) the notion of eigenmodes of the structure. They are either normal modes (defined by adding conservative conditions to the model) or complex modes (taking into account viscous damping for example) $[1,2]$. The linear theory of differential systems provides the response of the structure to an external elementary sinusoidal excitation in an interesting form: the full response is simply the superposition of the responses of each mode to the excitation. Such a formula is well known; this is the superposition formula which is the basis of modal synthesis [2, 3]. The notion of modal synthesis can be extended to the case of sub-structures by using linear operator theory $[3,4]$.

In the non-linear case, the notion of non-linear modes had been considered first. In the case of mathematically smooth non-linearities and for a finite number of degrees of freedom (d.o.f.) with particular polynomial non-linearities, Rosenberg and others first introduced natural modes [5] and then non-linear normal modes [6-9], and investigated their stability. Since then, many methods have been used to introduce modes (natural, non-linear, non-linear normal, minimal normal, non-linear similar normal, etc.) in the case of non-linear structures; methods derived from the works of Rosenberg [10-13]; a stroboscopic method [14]; methods based on averaging and modal truncation [15-17]; direct or geometrical methods for conservative systems [18-20] or in the Hamiltonian frame [21-25]; Padé approximation [26, 27]; multi-spectral, Volterra series and HFRFs $[28,29]$; integral transforms [30]; Lie series [31]; methods based on normal forms in the Hamiltonian [32-34] or general frame [35-40]; or methods using centre manifold theory [41-47] or amplitude equations [48]. Jézéquel and Lamarque extended the modal 
superposition starting from non-linear modes built via normal forms, for systems with a few d.o.f. and smooth non-linearities [49-51], even for the case of complex modes [52]; this method is valid for sufficiently small non-linear oscillations. The non-linear modes and generalized masses obtained depend on the amplitudes of the normal co-ordinates. They are built up in order to agree at best with a resonance equation. The modes do not always verify the reciprocity condition which exists in the linear case.

The question of non-linear modes is obviously related to the search for periodic solutions to non-linear dynamical systems and the study of their stability [52, 53]. It therefore involves a huge amount of literature dealing with numerous analytical methods, perturbation methods, and methods for bifurcation analysis for example references [32, 54-66].

In the field of stochastic behaviour, the question of non-linear modes has been examined already [67] and tools are available [68]. In the case of non-smooth non-linearities, only particular cases have been investigated; that is, normal modes for piecewise linear systems $[69,70]$. Sometimes, in order to deal with localized or weak non-linear non-smooth phenomena using linear methods, one can introduce a modified dissipation or stiffness matrix.

From the point of view of dynamics, vibro-impact systems have been thoroughly studied in the literature: global behaviours and periodic solutions have been investigated in the single-d.o.f. case (both analytically and numerically in references [71-74] or by means of a change of variables in reference [75]) and in the two-d.o.f. case (double impact oscillator in references $[76,77]$ or impact damper in references $[78,79])$. Singularities in the dynamics of such systems have been pointed out in references in [80-82], and some authors have examined the effect of dry friction on mechanical systems with impacts in [83-86]. General results for vibro-impact systems can also be found in references [87, 88]. Moreover, a modal approach has been introduced in reference [89] to deal with direct and inverse problems in discrete systems with impacts, based on the theory of non-linear normal modes (see reference [27]).

Nevertheless, to our knowledge, no attempt to build a modal superposition similar to the linear case exists in the case of hard non-smooth non-linearities such as friction or impact. The main aim of this work is to seek some answers to the question about possibility of building a modal superposition and a modal synthesis in the case of structures exhibiting a non-linearity of impact type. The problem is considered for the case of simple systems with one and two d.o.f.

In section 2, a single d.o.f. system is considered. Using a piecewise exact integration (2.1), the periodic responses under sinusoïdal excitation are studied (2.2). The building of a modal superposition in equation (2.3) is then tested by introducing successively a generalized eigenfrequency from free vibrations, a generalized mode and a generalized mass associated with forced oscillations.

In section 3, two-d.o.f. systems are considered. First the case of weak coupling and impact of a mass against a rigid stop is dealt with (3.1). Then the case of strong coupling is examined, again with impacts against a external rigid stop (3.2). The results obtained are applied to the case of direct impacts between two rigid solids (3.3). Finally, in section 4 conclusions are drawn on the relevance of the modal superposition formula obtained.

\section{SINGLE-DEGREE-OF-FREEDOM SYSTEM}

The system studied consists of a single-d.o.f. damped harmonic oscillator with a unilateral constraint, for which an impact law is defined (see Figure 1). The impact process 

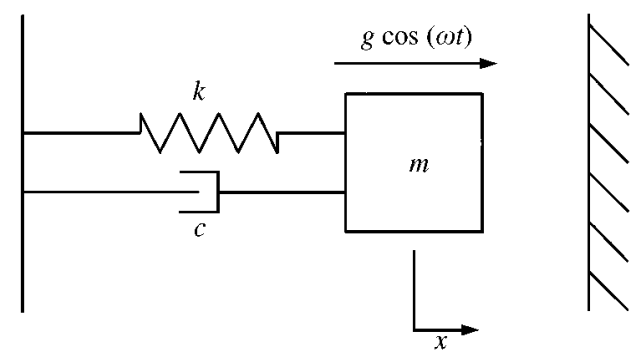

Figure 1. Single-degree-of-freedom system with $k=\omega_{1}^{2} m, c=a m, g=f m$.

is considered to be instantaneous and the behaviour of the system at the time of impact is described using the coefficient of restitution $e \in[0,1]$, characterizing the energy loss during impact. The equations governing the dynamics of the system are then

$$
\begin{aligned}
& \ddot{x}+a \dot{x}+\omega_{1}^{2} x=f \cos (\omega t), \\
& x(t) \leqslant x_{\max }, \\
& \dot{x}\left(t^{+}\right)=-e \dot{x}\left(t^{-}\right) \quad \text { if } x(t)=x_{\max } .
\end{aligned}
$$

If the velocity immediately before the impact at $\bar{t}$ is zero, several cases can occur: if at $\bar{t}$ the acceleration is negative, then the system is still described by equation (1) after the impact and the trajectory is tangent to the stop at $\bar{t}$. If, on the other hand, the acceleration is positive, then the system remains in contact with the stop for a non-zero time interval. It can be shown that sticking never occurs if $f<\omega_{1}^{2} x_{\max }$. In the following, it is assumed that the system's parameters satisfy this condition.

\subsection{ANALYTICAL SOLUTION}

The system with sinusoïdal forcing can be written:

$$
\begin{aligned}
& \ddot{x}+a \dot{x}+\omega_{1}^{2} x=f \cos (\omega t), \\
& \dot{x}\left(t^{+}\right)=-e \dot{x}\left(t^{-}\right) \quad \text { if } x(t)=x_{\max }, \\
& x(0)=x_{0}, \quad \dot{x}(0)=\dot{x}_{0} .
\end{aligned}
$$

Since this system is linear between two consecutive impacts, it is possible to determine a piecewise analytical form of the solution on $\mathbb{R}^{+}$. Setting $\tilde{\omega}_{1}=\sqrt{\omega_{1}^{2}-a^{2} / 4}$ and $\eta=a / 2 \tilde{\omega}_{1}$. $\forall k \in \mathbb{N}^{*}$, the solution on $\left[t_{k-1}, t_{k}\right]$ can be written in the form

$$
\begin{aligned}
x(t) & =\mathrm{e}^{-a t / 2}\left[A_{k} \cos \left(\tilde{\omega}_{1} t\right)+B_{k} \sin \left(\tilde{\omega}_{1} t\right)\right]+f_{1} \cos (\omega t)+f_{2} \sin (\omega t), \\
x\left(t_{k}^{+}\right) & =x\left(t_{k}^{-}\right)=x_{\max } \\
\dot{x}\left(t_{k}^{+}\right) & =-e \dot{x}\left(t_{k}^{-}\right)
\end{aligned}
$$


where $t_{0}=0$ and

$$
\begin{aligned}
& f_{1}=f \frac{\omega_{1}^{2}-\omega^{2}}{\left(\omega_{1}^{2}-\omega^{2}\right)^{2}+a^{2} \omega^{2}}, \\
& f_{2}=f \frac{a \omega}{\left(\omega_{1}^{2}-\omega^{2}\right)^{2}+a^{2} \omega^{2}} .
\end{aligned}
$$

From equations (3), the following recursive relation gives the values of the constants $A_{k}$ and $B_{k}$ :

$$
\begin{aligned}
& \left(\begin{array}{c}
A_{1} \\
B_{1}
\end{array}\right)=\left(\begin{array}{c}
x_{0}-f_{1} \\
\frac{\dot{x}_{0}-f_{2} \omega}{\tilde{\omega}_{1}}+\eta\left(x_{0}-f_{1}\right)
\end{array}\right), \\
& \left(\begin{array}{c}
A_{k+1} \\
B_{k+1}
\end{array}\right)=\left(\begin{array}{c}
A_{k} \\
B_{k}
\end{array}\right)+(1+e) u\left(A_{k}, B_{k}, t_{k}\right)\left(\begin{array}{c}
-\sin \left(\tilde{\omega}_{1} t_{k}\right) \\
\cos \left(\tilde{\omega}_{1} t_{k}\right)
\end{array}\right), \\
& u\left(A_{k}, B_{k}, t_{k}\right)=\left[\sin \left(\tilde{\omega}_{1} t_{k}\right)+\eta \cos \left(\tilde{\omega}_{1} t_{k}\right)\right] A_{k} \\
& +\left[-\cos \left(\tilde{\omega}_{1} t_{k}\right)+\eta \sin \left(\tilde{\omega}_{1} t_{k}\right)\right] B_{k} \\
& +\frac{\omega}{\tilde{\omega}_{1}} \mathrm{e}^{a t_{k} / 2}\left[f_{1} \sin \left(\omega t_{k}\right)-f_{2} \cos \left(\omega t_{k}\right)\right] .
\end{aligned}
$$

\subsection{PERIODIC SOLUTIONS}

Owing to the analytical form of the solution given by equations (3) and (4) it is possible to seek analytically a periodic solution, similar to that achieved in reference [72] or [71] for example. A solution of period $n T$ with $k$ impacts per cycle is here called $(n, k)$-periodic, where $T=2 \pi / \omega$ is the period of the external excitation.

\subsection{1. (n, 0)-periodic solutions}

The simplest case consists of looking for $n T$-periodic solutions which never impact against the stop. Such a case implies $n=1$ and the initial conditions leading to a (1,0)-periodic solution are

$$
\begin{aligned}
& x_{0}=f_{1}, \\
& \dot{x}_{0}=f_{2} \omega .
\end{aligned}
$$

Setting:

$$
\begin{aligned}
& \omega_{+}=\sqrt{\tilde{\omega}_{1}^{2}-\frac{a^{2}}{4}+\sqrt{\frac{f^{2}}{x_{\max }^{2}}-a^{2} \tilde{\omega}_{1}^{2}}}, \\
& \omega_{-}=\sqrt{\tilde{\omega}_{1}^{2}-\frac{a^{2}}{4}-\sqrt{\frac{f^{2}}{x_{\max }^{2}}-a^{2} \tilde{\omega}_{1}^{2}}}
\end{aligned}
$$


and as it is assumed that $f<\omega_{1}^{2} x_{\text {max }}$, it can be shown that $(n, 0)$-periodic solutions exist if and only if $\omega \leqslant \omega_{-}$or $\omega \geqslant \omega_{+}$.

The stability of these periodic solutions can be determined using a Poincaré map defined by a constant phase plane $Z=T$ in the co-ordinates $(X, Y, Z)=(x, \dot{x}, t \bmod T)$. As pointed out in reference [80], such a mapping is not everywhere continuous nor differentiable. Therefore, for each periodic solution corresponding to a fixed point of the Poincare map where it is continuously differentiable, the stability can be investigated. It can be shown that $(1,0)$-periodic solutions are always stable.

\subsection{2. (n, 1)-periodic solutions}

Similar to section 2.2.1, $n T$-periodic solutions with one impact per cycle can be sought by using equations (3) and (4). In this case, the impact time can be analytically determined, and the initial conditions leading to $(n, 1)$-periodic solutions are then given by

$$
\begin{aligned}
& x_{0}=A_{1}\left(t_{1}\right)+f_{1}, \\
& \dot{x}_{0}=\tilde{\omega}_{1}\left[B_{1}\left(t_{1}\right)-\eta A_{1}\left(t_{1}\right)\right]+\omega f_{2},
\end{aligned}
$$

where $A_{1}\left(t_{1}\right)$ and $B_{1}\left(t_{1}\right)$ depend analytically on the system's parameters. An example of $(1,1)$-periodic solution is shown in Figure 2.

As in section 2.2.1, the Poincare map can be used (when it is defined and of class $\mathrm{C}^{1}$ locally) in order to determine the type of the periodic solutions obtained (see in Figure 3 ). The Jacobian matrix can be calculated analytically from the analytical form of the Poincare map by taking into account the influence of the partial derivatives of the impact time $t_{1}$ with respect to the initial conditions.

It can be shown that such a stability study is valid only if $\omega \neq \omega_{+}$and $\omega \neq \omega_{-}$for in that case the Poincaré map is not differentiable at the fixed point considered.

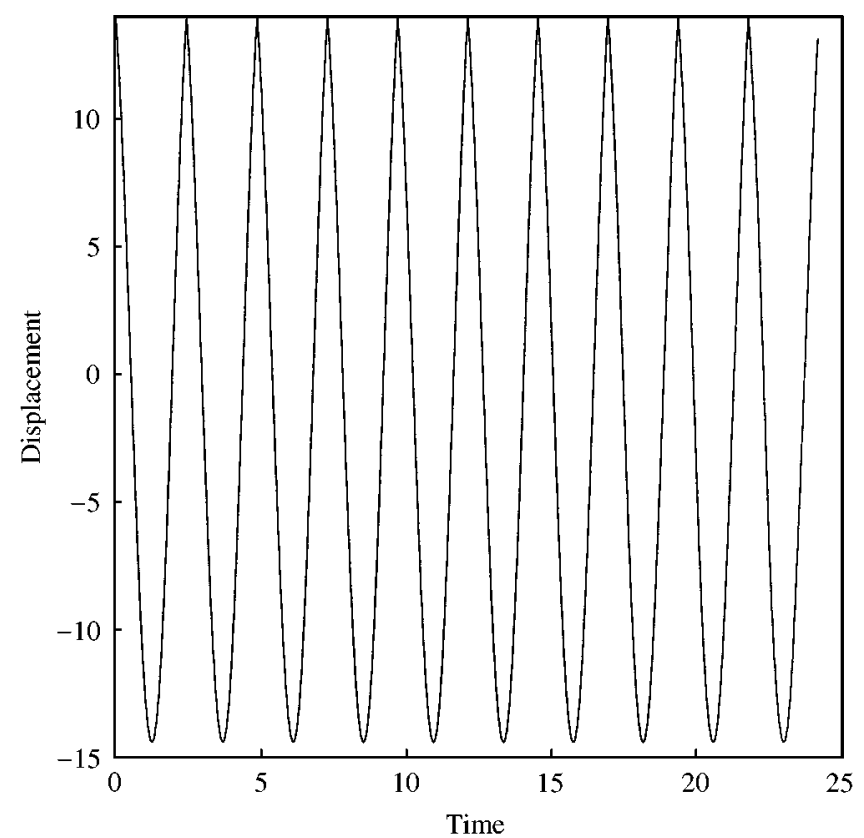

Figure 2. (1, 1)-periodic solution to the system (2) for $\omega=2 \cdot 6, x_{0}=13 \cdot 32968$ and $\dot{x}_{0}=19 \cdot 36619$. 


\subsection{3. (n, 2)-periodic solutions}

The method for seeking $n T$-periodic solutions with two impacts per cycle is identical to the case with one impact per cycle, with a new unknown due to the second impact time $t_{2}$. By characterizing the periodicity of the solution and by using the analytical form (3), (4) a system of two non-linear equations with two unknowns $\left(t_{1}, t_{2}\right)$ can be obtained which can for example be solved using Newton's method. Once the value of $t_{1}$ and $t_{2}$ are known, the initial conditions of the system are given by

$$
\begin{aligned}
& x_{0}=A_{1}\left(t_{1}, t_{2}\right)+f_{1}, \\
& \dot{x}_{0}=\tilde{\omega}_{1}\left(B_{1}\left(t_{1}, t_{2}\right)-\eta A_{1}\left(t_{1}, t_{2}\right)\right)+\omega f_{2} .
\end{aligned}
$$

Figures 4-6 show three examples of $(n, 2)$-periodic solutions that can be obtained analytically.

As in the case of $(n, 1)$-periodic solutions, the stability of the $(n, 2)$-periodic solutions can be studied by using the Poincaré map: when $\omega \notin\left\{\omega_{+}, \omega_{-}\right\}$, it is possible to calculate analytically the Jacobian matrix of the Poincare map, and its eigenvalues determine the stability or instability of the periodic solution.

\subsection{MODAL SUPERPOSITION}

\subsubsection{Free oscillations}

The free oscillations of the system are described by the following equation:

$$
\ddot{x}+a \dot{x}+\omega_{1}^{2} x=0
$$

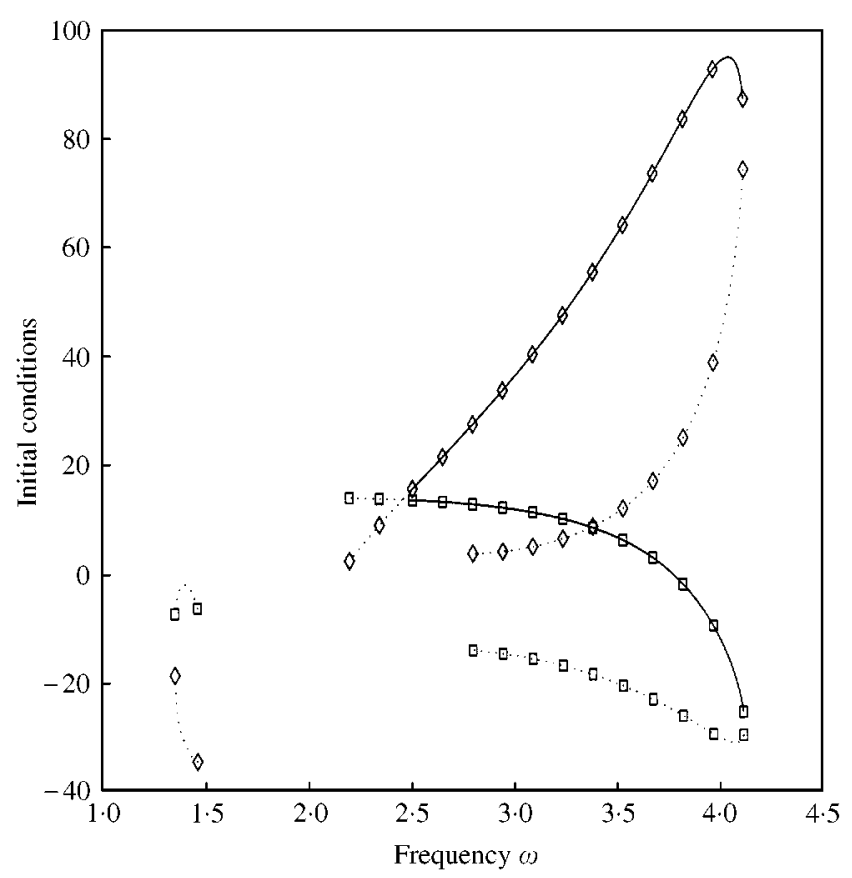

Figure 3. Existence of $(1,1)$ periodic solutions. Stable (square: $x_{0}$, diamond: $\left.\dot{x}_{0}\right)$ and unstable (dotted curve) solutions. 


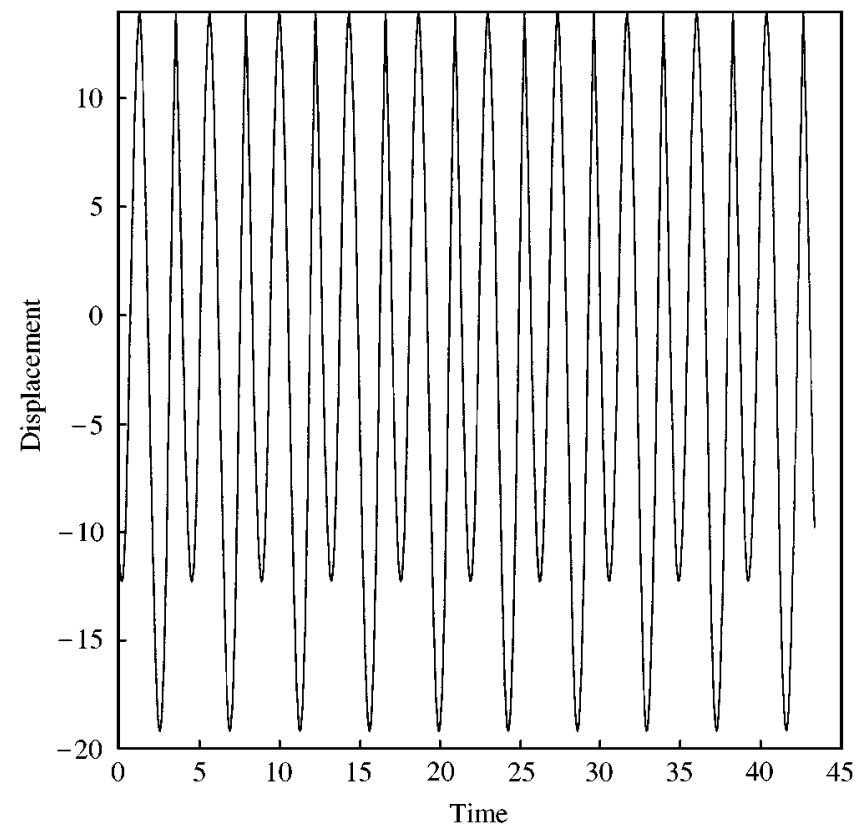

Figure 4. (1, 2)-periodic solution for $\omega=1 \cdot 45, x_{0}=-9 \cdot 89493$ and $\dot{x}_{0}=-20 \cdot 71102$.

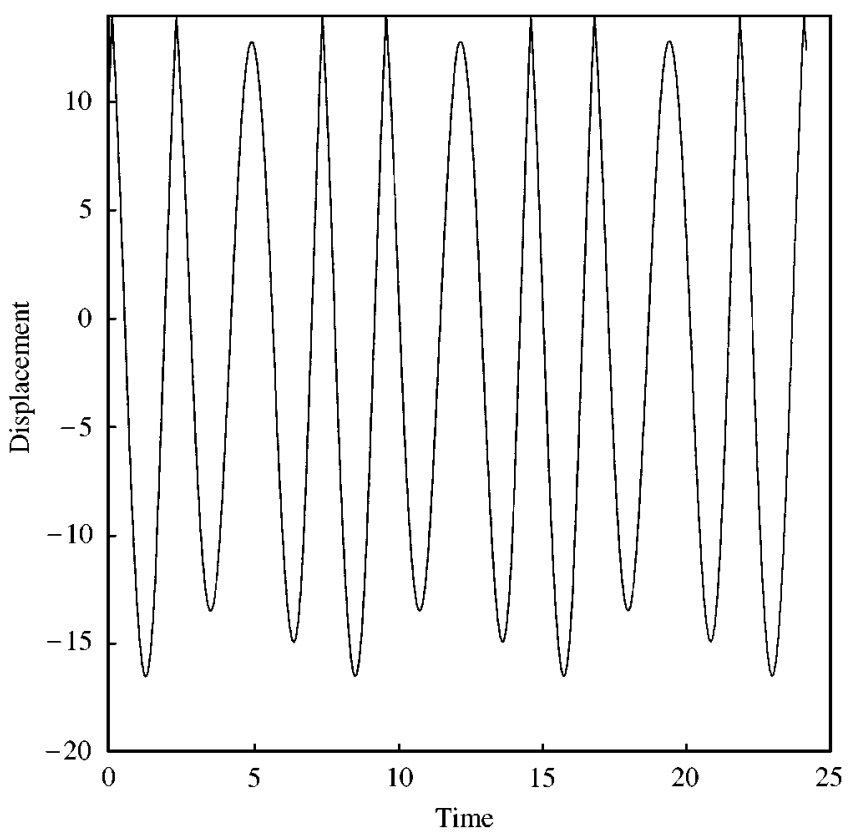

Figure 5. (3, 2)-periodic solution for $\omega=2 \cdot 6, x_{0}=9 \cdot 77211$ and $\dot{x}_{0}=35 \cdot 46891$.

$$
\begin{aligned}
& \dot{x}\left(t^{+}\right)=-e \dot{x}\left(t^{-}\right) \quad \text { if } x(t)=x_{\max }, \\
& x(0)=x_{0}, \quad \dot{x}(0)=\dot{x}_{0} .
\end{aligned}
$$

The solution of this equation can also be piecewise analytically written: the expression is identical to equation (3), the constants of integration being given by equation (4), with $f_{1}=f_{2}=0$. 


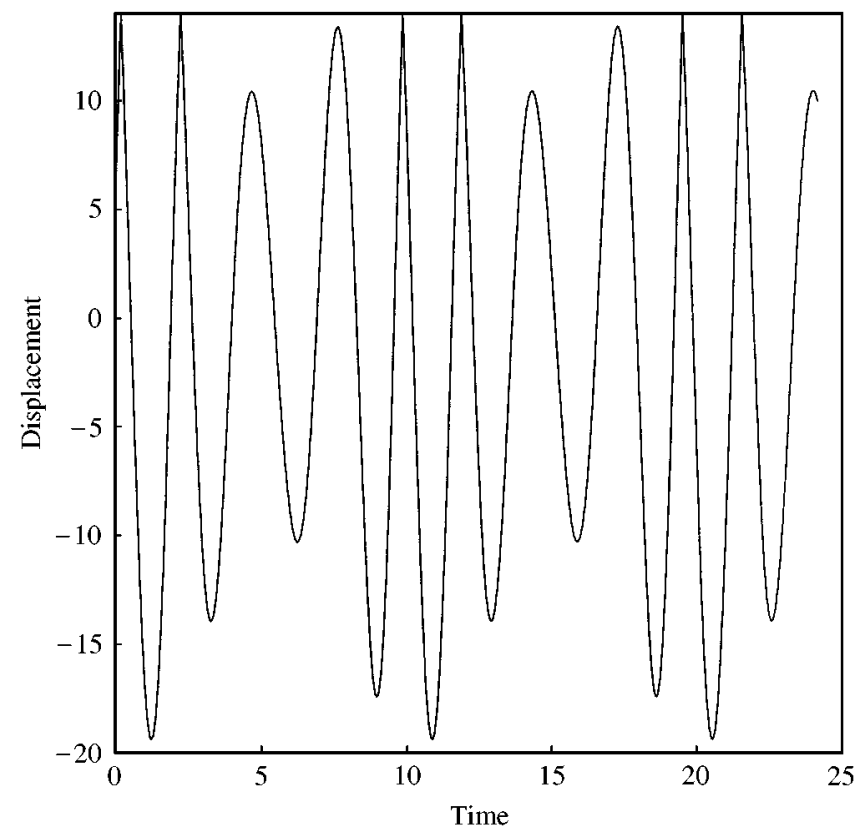

Figure 6. (4, 2)-periodic solution for $\omega=2 \cdot 6, x_{0}=4 \cdot 76445$ and $\dot{x}_{0}=47 \cdot 91445$.

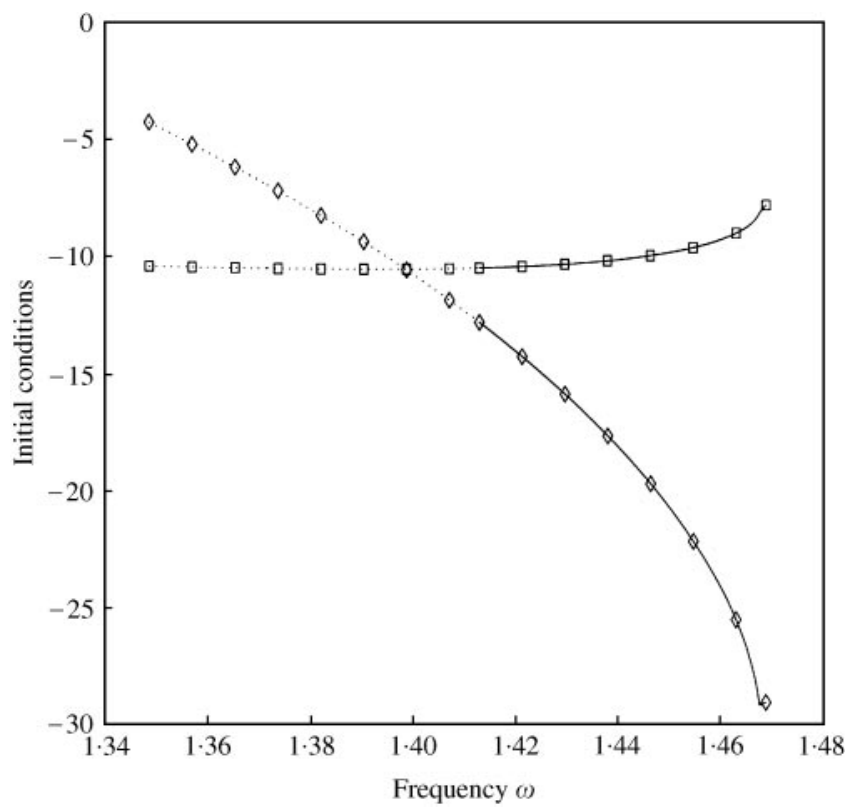

Figure 7. Existence of $(1,2)$ periodic solutions. Stable (square: $x_{0}$, diamond: $\left.\dot{x}_{0}\right)$ and unstable (dotted curve) solutions.

If the equilibrium is a position that the system can physically reach, namely if $x_{\max }>0$, and if $a \neq 0$, it can be shown that the system (6) has a finite number $K$ of impacts. This result is interesting from the modal point of view; it means that the free oscillations of the system are governed by the frequency $\omega_{1}$, except for a bounded time span. Hence, it will be considered later on that the natural frequency of the system is $\omega_{1}$. 

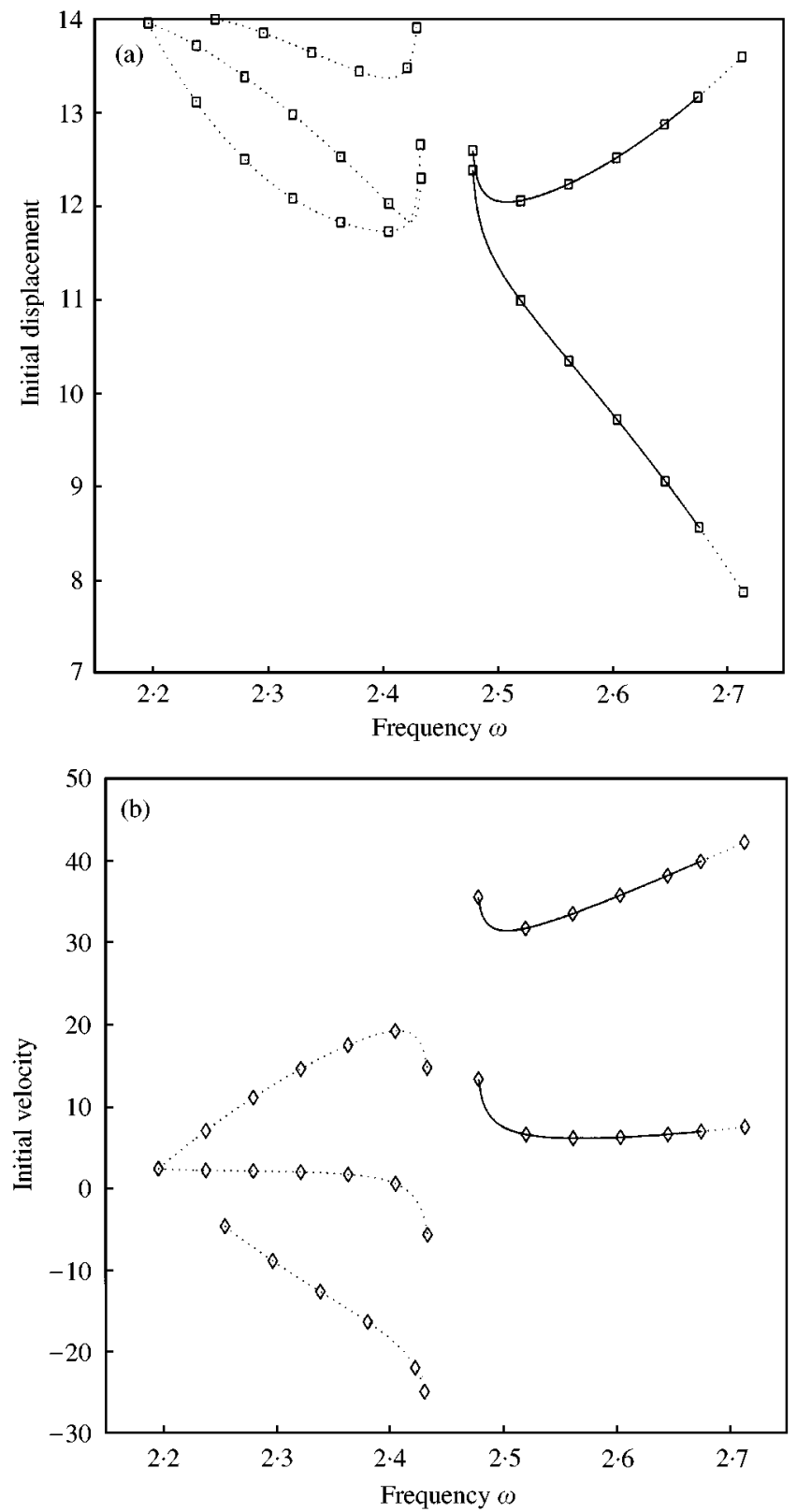

Figure 8. Existence of (3,2)-periodic solutions: stable (solid curve) and unstable solutions (dotted curve): (a) initial displacements; (b) initial velocities.

Remark. When $a=0$, the number of impacts is infinite and $\lim _{k \rightarrow+\infty} t_{k+1}-t_{k}=2 \pi / \tilde{\omega}_{1}$. If $x_{\max }=0$, there also is an infinite number of impacts and $t_{k+1}-t_{k}=\pi / \tilde{\omega}_{1}, \forall k$. Finally, if $x_{\text {max }}<0$ then $\lim _{k \rightarrow+\infty} t_{k+1}-t_{k}=0$.

\subsubsection{Generalized mass and modal superposition}

In this section, it is intended to establish a modal superposition formula for the irregular non-linear model previously introduced. The case $x_{\max }>0$ is considered for which the 

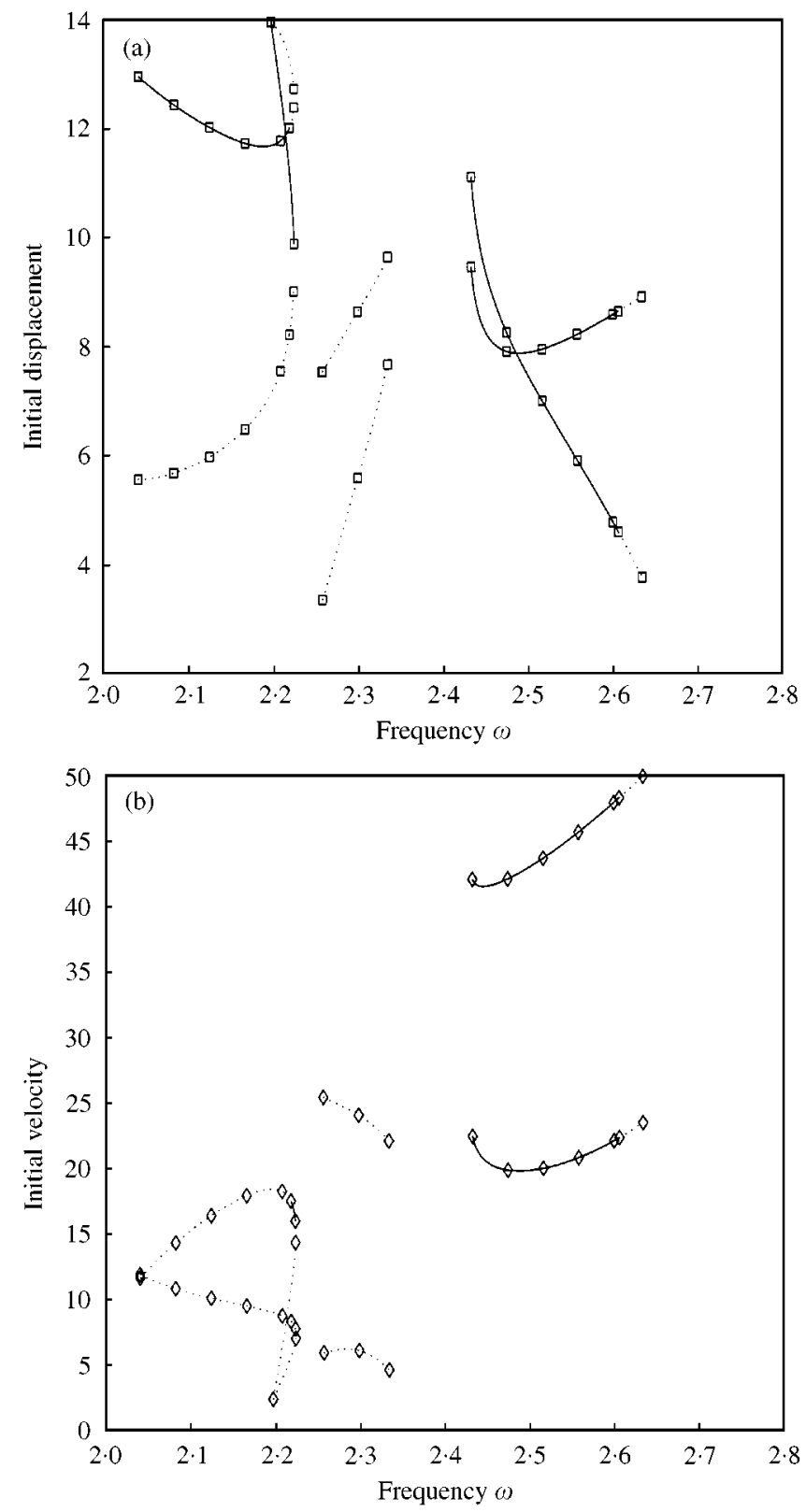

Figure 9. Existence of (4, 2)-periodic solutions: stable (solid curve) and unstable solutions (dotted curve): (a) initial displacements; (b) initial velocities.

natural frequency is $\omega_{1}$, and an attempt is made to obtain a modal superposition formula similar to the linear case, connecting the $n$th harmonic amplitude of the response to the amplitude of the forcing.

If a $(n, k)$-periodic solution is considered, i.e. a solution with period $n T$ and $k$ impacts per cycle. Due to the $n T$-periodicity of the response, the Fourier coefficients can be defined; for 
all $j \in \mathbb{Z}$ we have

$$
\begin{aligned}
c_{j}(\omega)= & \frac{1}{n T} \int_{0}^{n T} x(t) \exp \left(-\mathrm{i} \frac{j}{n} \omega t\right) \mathrm{d} t \\
= & \frac{1}{n T}\left[\int_{0}^{t_{1}} x(t) \exp \left(-\mathrm{i} \frac{j}{n} \omega t\right) \mathrm{d} t+\sum_{m=1}^{k-1} \int_{t_{m}}^{t_{m+1}} x(t) \exp \left(-\mathrm{i} \frac{j}{n} \omega t\right) \mathrm{d} t\right. \\
& \left.+\int_{t_{k}}^{n T} x(t) \exp \left(-\mathrm{i} \frac{j}{n} \omega t\right) \mathrm{d} t\right] .
\end{aligned}
$$

$x(t)$ is known to be piecewise via equation (3). These $k+1$ integrals can then be calculated analytically. If $k \neq 0$ define

$$
\begin{aligned}
H_{n, k}^{j}(\omega)= & \frac{2 \tilde{\omega}_{1}}{n T}\left\{B_{1}+A_{k+1} \mathrm{e}^{-a n T / 2} \sin \left(n \tilde{\omega}_{1} T\right)-B_{k+1} \mathrm{e}^{-a n T / 2} \cos \left(n \tilde{\omega}_{1} T\right)\right. \\
& +\left(\eta+\mathrm{i} \gamma_{j}\right)\left[A_{1}-A_{k+1} \mathrm{e}^{-a n T / 2} \cos \left(n \tilde{\omega}_{1} T\right)-B_{k+1} \mathrm{e}^{-a n T / 2} \sin \left(n \tilde{\omega}_{1} T\right)\right] \\
& \left.+(1+e) \sum_{m=1}^{k} \mathrm{e}^{-a t_{m} / 2} \exp \left(-\mathrm{i} \frac{j}{n} \omega t_{m}\right) u\left(A_{m}, B_{m}, t_{m}\right)\right\},
\end{aligned}
$$

where $\gamma_{j}=j \omega / n \tilde{\omega}_{1}$, and if $k=0, H_{n, 0}^{j}(\omega)=0$. Then

$$
c_{j}(\omega)=\frac{f_{1}-\mathrm{i} f_{2}}{2} \delta_{j}^{n}+\frac{f_{1}-\mathrm{i} f_{2}}{2} \delta_{j}^{-n}+\frac{H_{n, k}^{j}(\omega)}{2} .
$$

Let

$$
\Delta I_{1}(\omega)=\omega_{1}^{2}-\omega^{2}+a \mathrm{i} \omega .
$$

The $n$th Fourier coefficient is then given by

$$
c_{n}(\omega)=\frac{f_{1}-\mathrm{i} f_{2}}{2}+\frac{H_{n, k}^{n}(\omega)}{2 \Delta I_{1}(\omega)}=\frac{f+H_{n, k}^{n}(\omega)}{2 \Delta I_{1}(\omega)} .
$$

Let the modal mass $m_{n, k}$ be:

$$
m_{n, k}=\frac{1}{1+H_{n, k}^{n}(\omega) / f} .
$$

Because the free damped steady state oscillations are linear oscillations, the generalized mode is here represented by the scalar 1 . The $n$th harmonic amplitude can then be written in the form

$$
\mathscr{A}_{n}(\omega)=\sqrt{2\left(\left|c_{n}\right|^{2}+\left|c_{-n}\right|^{2}\right)}=2\left|c_{n}\right|=\left|\frac{f}{m_{n, k} \Delta I_{1}(\omega)}\right| .
$$

This is a modal superposition formula connecting the $n$th harmonic amplitude of the forced response to the amplitude of the forcing via the free response. This formula is similar to the formula that obtained in the linear case, but the mass (which should be equal to 1) is replaced by a modal mass (11).

Due to this definition, the mass is a complex: to give it a more physical meaning, it is necessary to consider its module. Thus, the unitary mass system with impact is modelled and, subjected to a sinusoïdal forcing of frequency $\omega$, like a linear system without impact of 
mass $\left|m_{n, k}\right|$, subjected to the same forcing. This modelling holds in terms of spectral amplitude for a $(n, k)$-periodic response: the spectral amplitude is the same one for both systems.

\section{Remark.}

- In the case of a $n T$-periodic response without any impacts, it was seen that only the case $n=1$ was possible. Thus, $m_{1,0}=1$ which is coherent since in this case the classical modal superposition formula applies and gives $\mathscr{A}_{1}(\omega)=\left|f / \Delta I_{1}(\omega)\right|$.

- There is no longer a unique modal superposition formula as in the linear case, but an infinity a priori; it depends on the period and number of impacts per cycle of the periodic solution.

- Preceding calculations give access to the whole Fourier series of a $(n, k)$-periodic response.

- An analytical expression of the module of the modal mass is obtained if an only if $k=1$, i.e., when the periodic solution has only one impact by period. Setting

$$
\begin{aligned}
X_{1}= & \frac{2(1+e) \omega}{n f T} \frac{-1+2 \mathrm{e}^{a n T / 2} \cos \left(n \tilde{\omega}_{1} T\right)-\mathrm{e}^{a n T}}{e+\mathrm{e}^{a n T / 2}\left\{(1-e) \cos \left(n \tilde{\omega}_{1} T\right)+(1+e) \eta \sin \left(n \tilde{\omega}_{1} T\right)\right\}-\mathrm{e}^{a n T}} \\
& \times\left[f_{1} \sin \left(\omega t_{1}\right)-f_{2} \cos \left(\omega t_{1}\right)\right]
\end{aligned}
$$

for the modal mass:

$$
m_{n, 1}=\frac{1}{1+X_{1} \mathrm{e}^{-\mathrm{i} \omega t_{1}}}
$$

Since $t_{1}$ is analytically known in the case of a $(n, 1)$-periodic response, the modal mass $\left|m_{n, 1}\right|$ can be expressed analytically as a function of the parameters of the system.

\subsubsection{Examples of modal superposition}

It is now possible to show some applications of the modal superposition formula previously established, using the analytical search for periodic solutions carried out in section 2.2. First consider the following set of parameters for the system: $\omega_{1}=2 \cdot 5$, $a=0.05, x_{\max }=14, e=0.9$ and $f=20$. Note that for these values of parameters $\omega_{1}^{2} x_{\max }>f$ and $e>0$, so that sticking never occurs.

Figures 10 outlines an important difference compared to the linear case: the peak of amplitude corresponding to resonance does no longer exist around $\omega_{1}$. Consequently, for $\omega$ close to the natural frequency of the system, the modal mass is the largest because there is no resonance between the external forcing and the system. The maximum amplitude occurs at a frequency higher than the natural frequency, but the maximum reached is much weaker than in the linear case; there is not a true peak of amplitude in the usual sense.

In addition, the modal superposition formula previously established enables the amplitude of $n$th harmonic of a solution, whose period is $n T$ to be computed. In the linear case (without impact), this is sufficient to know the whole spectral response of the system: only $n=1$ occurs and the Fourier coefficients are all zero except the first one. In this case, it is no longer sufficient, for the occurrence of impacts leads to periodic solutions with many harmonics, and the $n$th harmonic amplitude can be a poor approximation to the spectral amplitude of the response. For example, Figure 12 shows that the second-harmonic amplitude for a $T$-periodic solution can be the largest one. In the same way, the Fourier coefficient $c_{0}$ can become large, can be seen in Figure 10. Table 1 summarizes the difference 


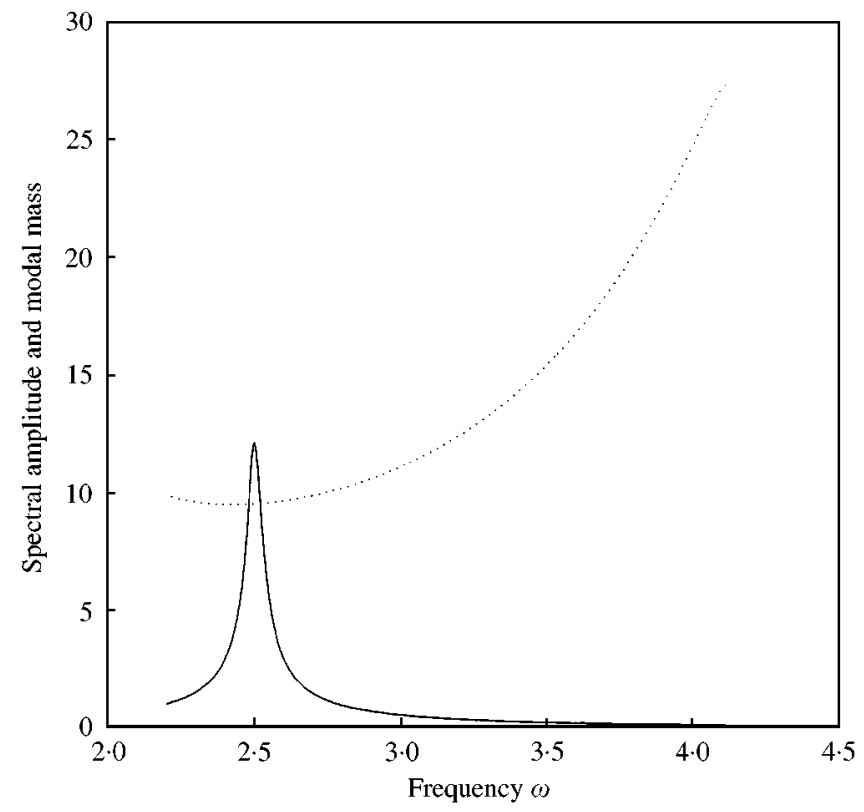

Figure 10. First-harmonic amplitude (dotted curve) and modal mass (solid curve) of the (1, 1)-periodic solutions.

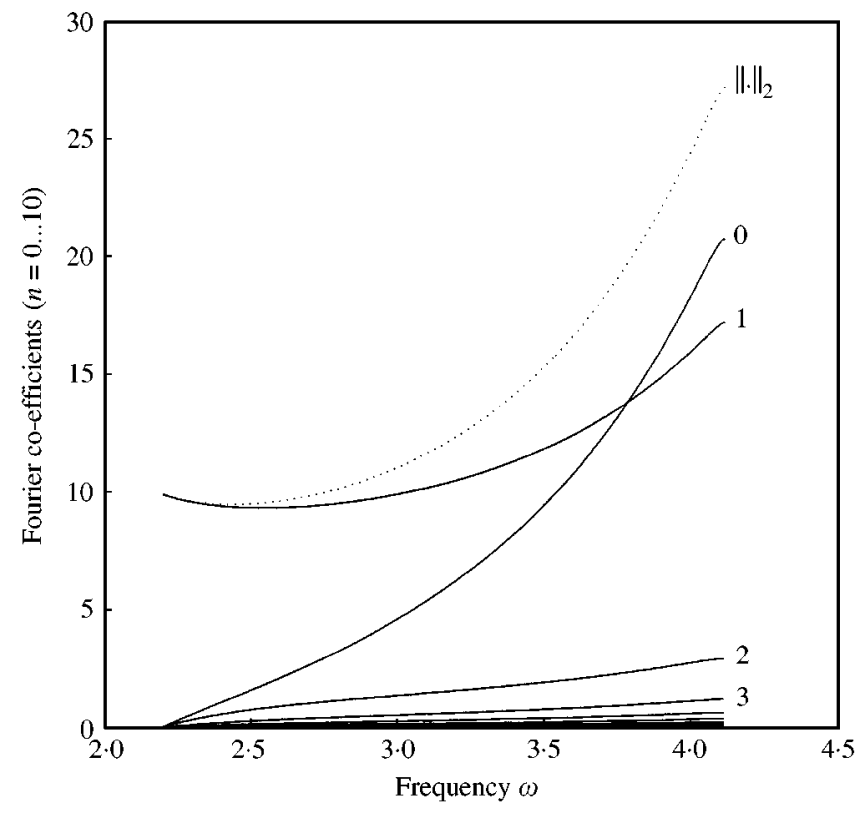

Figure 11. Fourier coefficients of the $(1,1)$-periodic responses.

between the spectral amplitude and the $n$th harmonic amplitude for various periodic solutions.

For this example, a modal superposition formula can be built and is worthwhile as long as $\omega$ remains close to the "primary resonance". Nevertheless, this frequency area does not always correspond to the largest amplitudes. 


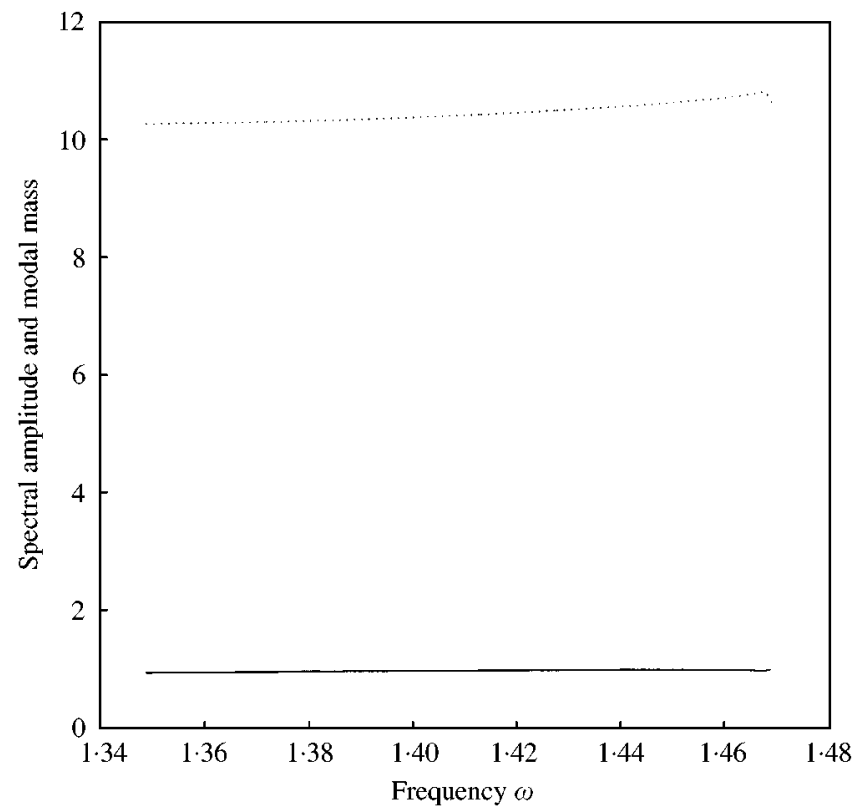

Figure 12. First-harmonic amplitude (dotted curve) and modal mass (solid curve) of the (1, 2)-periodic solutions.

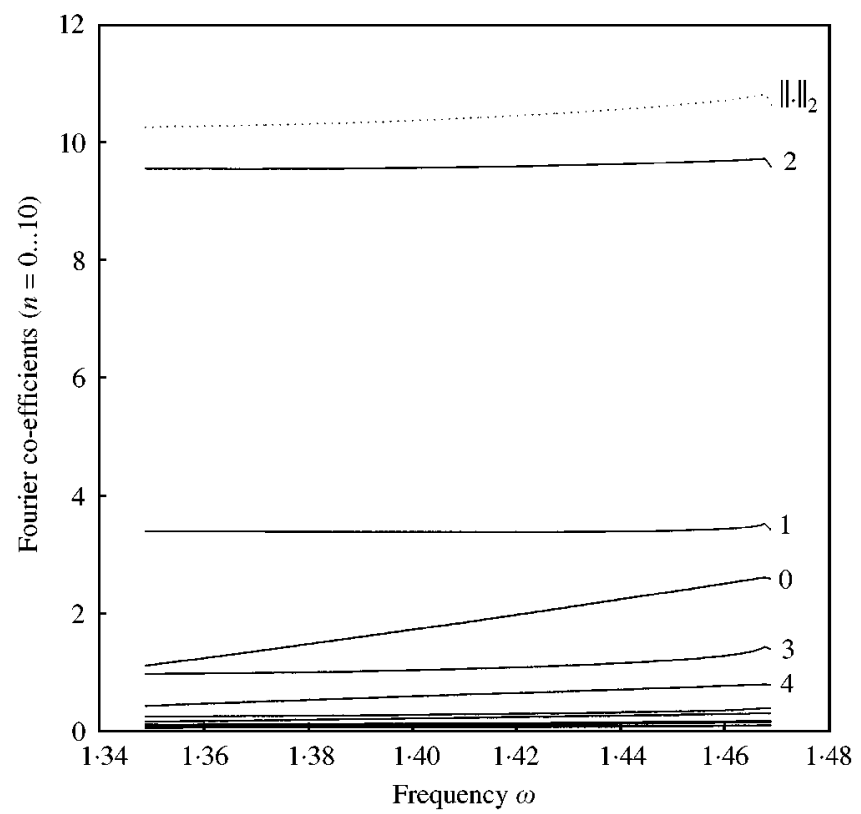

Figure 13. Fourier coefficients of the $(1,2)$-periodic responses.

An example showing a spectral behaviour different from the preceding one can now be considered. In this case $\omega_{1}=1, a=0.02, x_{\max }=1, e=0.9$ and $f=20$ (sticking to the stop may then occur, but only responses without sticking will be considered).

In Figure 18 two important characteristics can be seen. First of all, and contrary to the previous example, the response exhibits a true peak of spectral amplitude similar to the 


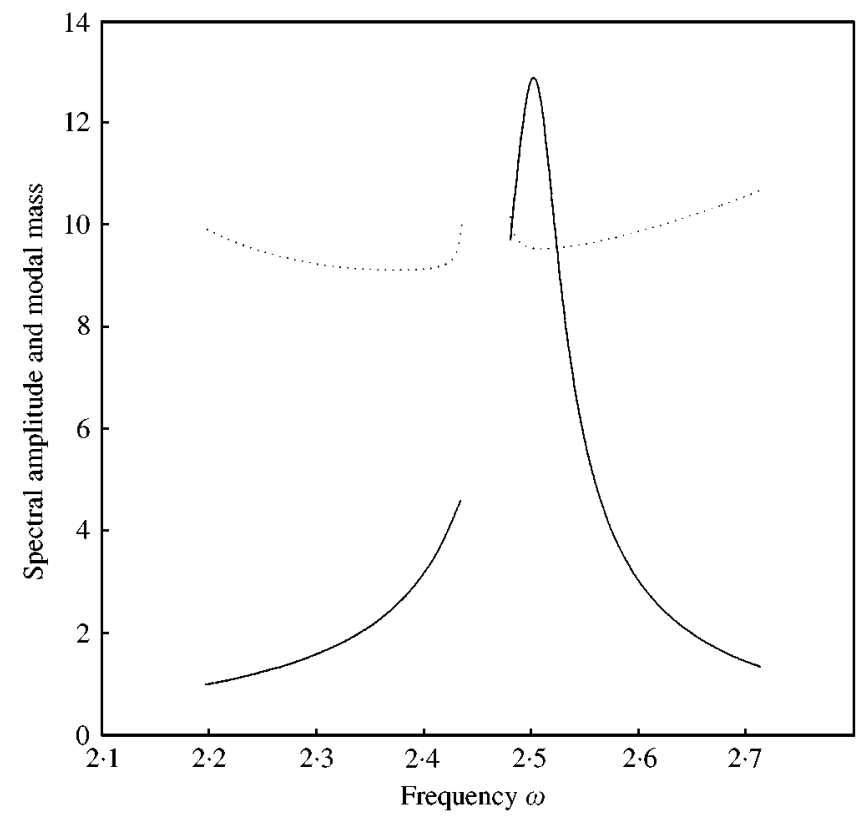

Figure 14. Third-harmonic amplitude (dotted curve) and modal mass (solid curve) of the (3,2)-periodic solutions.

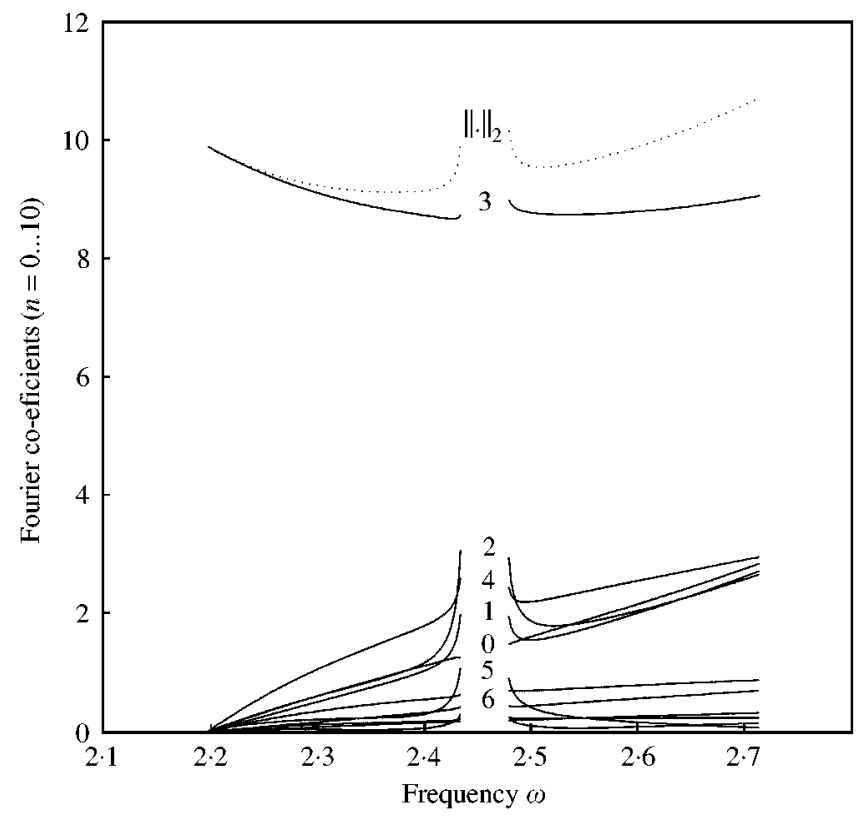

Figure 15. Fourier coefficients of the (3, 2)-periodic responses.

resonance peaks observed in linear systems. However, the peak does not occur at the natural frequency $\omega_{1}$, but at $\omega \approx 2 \omega_{1}$. Furthermore, the approximation to the whole amplitude by the amplitude of first harmonic is very rough: resonance occurs through the Fourier coefficient $c_{0}$ of the solution, and the remainder of the harmonics are negligible in the neighbourhood of the peak. 


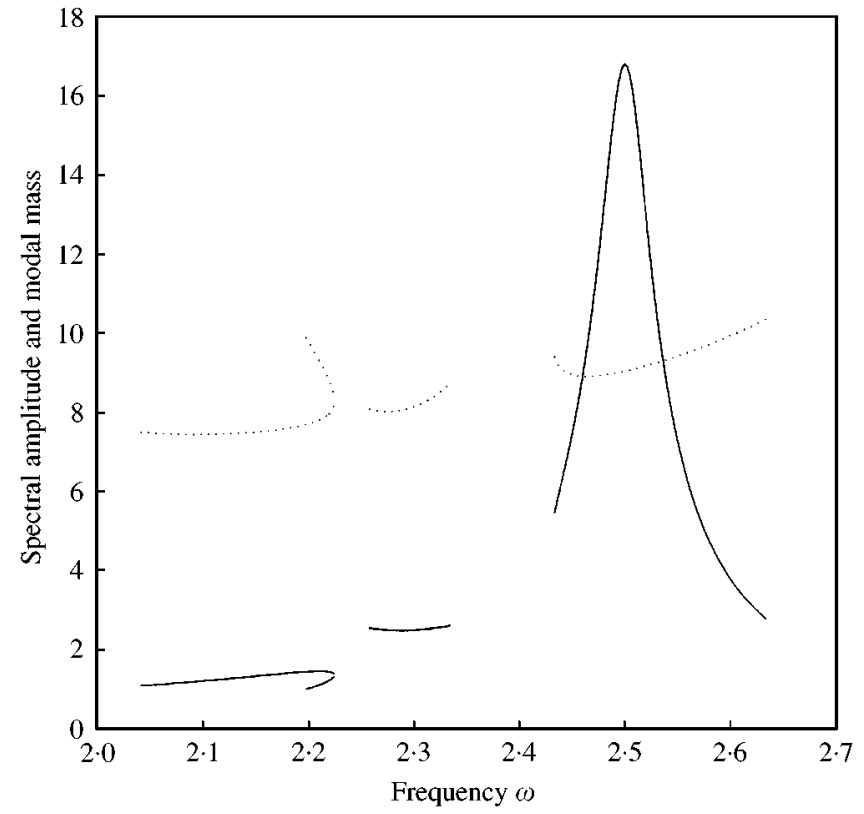

Figure 16. Fourth-harmonic amplitude (dotted curve) and modal mass (solid curve) of the (4, 2)-periodic solutions.

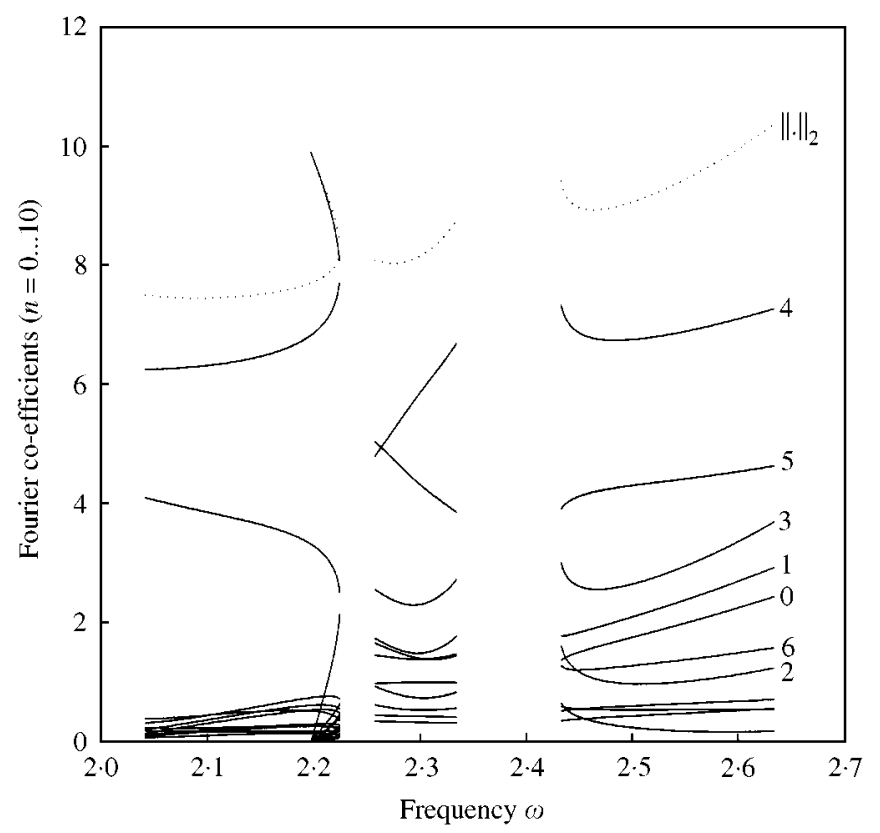

Figure 17. Fourier coefficients of the $(4,2)$-periodic responses.

The last two examples have shown that the building of a modal superposition formula similar to the linear case comes up against two major difficulties. On one hand, the multiplicity of types of periodic solutions prevents a unique formula which holds for any case: it is necessary to know a priori the period of the response and the number of impacts per cycle. On the other hand, taking into account only one harmonic in this superposition 


\section{TABLE 1}

Difference between Fourier amplitude and nth harmonic amplitude

\begin{tabular}{ccc}
\hline $\begin{array}{c}\text { Periodic } \\
\text { solution }\end{array}$ & $\begin{array}{c}\text { Difference (\%) } \\
\text { stable or unstable solution }\end{array}$ & $\begin{array}{c}\text { Difference (\%) } \\
\text { stable solution }\end{array}$ \\
\hline$(1,1)$ & $36 \cdot 65$ & $36 \cdot 65$ \\
$(1,2)$ & $67 \cdot 89$ & $67 \cdot 89$ \\
$(3,2)$ & $15 \cdot 56$ & $13 \cdot 96$ \\
$(4,2)$ & $40 \cdot 89$ & $28 \cdot 96$ \\
\hline
\end{tabular}

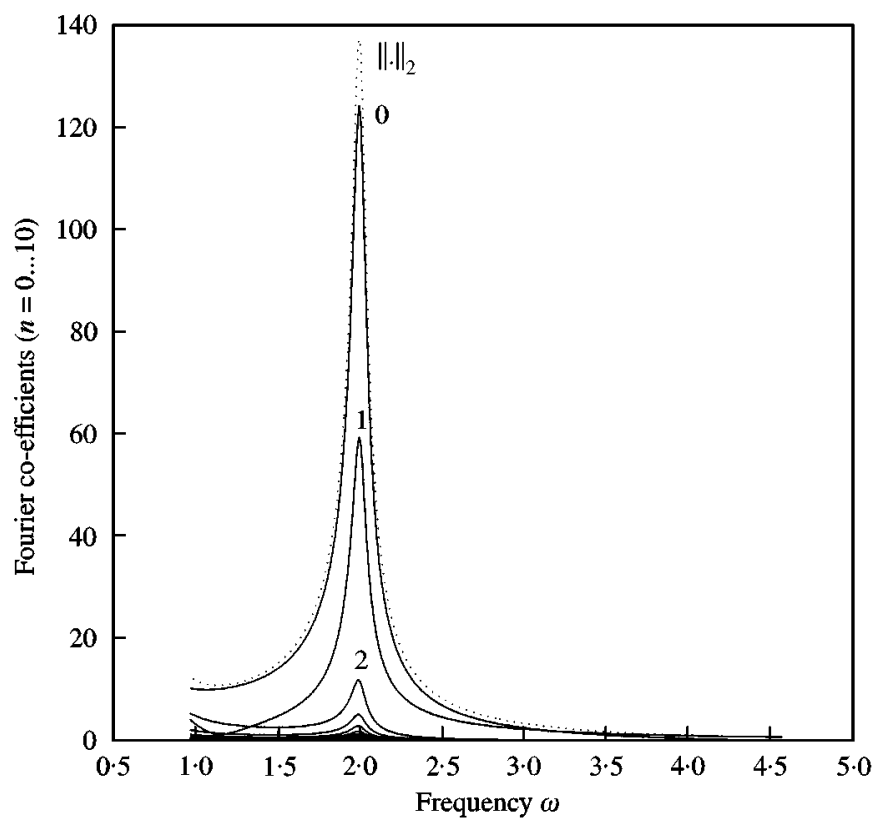

Figure 18. Fourier coefficients of the $(1,1)$-periodic responses.

may not be sufficient any more; it would be necessary to include at least the four first harmonics in the formula in order to get a closer approximation in the examples shown.

\section{TWO-DEGREE-OF-FREEDOM SYSTEMS}

This section deals with a system with two d.o.f., one of them being constrained by a stop:

$$
\begin{aligned}
& \ddot{x}+a \dot{x}+\omega_{1}^{2} x+k_{1} y=f_{1} \cos (\omega t), \\
& \left.\left.x \in \mathscr{C}^{0}\left(\mathbb{R}^{+},\right]-\infty, x_{\max }\right]\right), \\
& x(t)=x_{\max } \Rightarrow \dot{x}\left(t^{+}\right)=-e \dot{x}\left(t^{-}\right), \\
& \ddot{y}+a \dot{y}+\omega_{2}^{2} y+k_{2} x=f_{2} \cos (\omega t), \\
& y \in \mathscr{C}^{1}\left(\mathbb{R}^{+}, \mathbb{R}\right) .
\end{aligned}
$$


This system can be written in the form

$$
\ddot{\mathbf{X}}+\mathbf{A} \dot{\mathbf{X}}+\mathbf{K X}=\mathbf{F} \cos (\omega t)+\text { impact }
$$

with

$$
\mathbf{X}=\left(\begin{array}{l}
x \\
y
\end{array}\right), \quad \mathbf{A}=\left(\begin{array}{ll}
a & 0 \\
0 & a
\end{array}\right), \quad \mathbf{K}=\left(\begin{array}{cc}
\omega_{1}^{2} & k_{1} \\
k_{2} & \omega_{2}^{2}
\end{array}\right), \quad \mathbf{F}=\left(\begin{array}{l}
f_{1} \\
f_{2}
\end{array}\right)
$$

and the term " + impact" represents the constraint induced on $x$ by the occurrence of impacts.

The model just introduced is a general mathematical model. It will be studied in three stages: first of all, consider the case $k_{1}=0$ (which will be referred to as "weak coupling") for which the study is very close to the single-degree-of-freedom case in term of periodic solutions: it will give an initial outline of modal superposition with two d.o.f.

The general case where $k_{1} \neq 0$ (referred to as "strong coupling") will then be studied, for which the modal superposition is similar to the case $k_{1}=0$, except that the search for periodic solutions becomes more complex. Finally it can be seen how the modal superposition can be written in the case of two rigid bodies colliding.

Note, initially, that the first case is a mathematical model which cannot easily be expressed in mechanical terms. Indeed, the action-reaction principle prevents $x$ from acting on $y$ without $y$ acting on $x$. Therefore, it is necessary to consider carefully the physical conclusions that could be drawn from this model.

\subsection{WEAK COUPLING}

System (14), the special case where $k_{1}=0$, which is close to the single-d.o.f. case previously studied can be dealt with.

\subsubsection{Analytical solution of the system}

3.1.1.1. Decoupling equations. The system (14) can be decoupled in order to obtain explicit solutions for $x$ and $y$. If

$$
v_{2}=\frac{k_{2}}{\omega_{1}^{2}-\omega_{2}^{2}} \quad \text { and } P=\left(\begin{array}{ll}
1 & 0 \\
v_{2} & 1
\end{array}\right)
$$

and pre-multiply the system 14 without impacts by $P^{-1}$, a system in the new co-ordinates $\left(\begin{array}{l}x_{1} \\ x_{2}\end{array}\right)=P^{-1} X$ is obtained:

$$
\begin{aligned}
& \ddot{x}_{1}+a \dot{x}_{1}+\omega_{1}^{2} x_{1}=f^{1} \cos (\omega t), \\
& \ddot{x}_{2}+a \dot{x}_{2}+\omega_{2}^{2} x_{2}=f^{2} \cos (\omega t),
\end{aligned}
$$

where

$$
\begin{aligned}
& f^{1}=f_{1}, \\
& f^{2}=-v_{2} f_{1}+f_{2} .
\end{aligned}
$$


Written in that form, the system is easy to solve and as long as there is no impact:

$$
\begin{aligned}
& x_{1}(t)=\mathrm{e}^{-a t / 2}\left[A^{1} \cos \left(\tilde{\omega}_{1} t\right)+B^{1} \sin \left(\tilde{\omega}_{1} t\right)\right]+f_{1}^{1} \cos (\omega t)+f_{2}^{1} \sin (\omega t), \\
& x_{2}(t)=\mathrm{e}^{-a t / 2}\left[A^{2} \cos \left(\tilde{\omega}_{2} t\right)+B^{2} \sin \left(\tilde{\omega}_{2} t\right)\right]+f_{1}^{2} \cos (\omega t)+f_{2}^{2} \sin (\omega t),
\end{aligned}
$$

where $\omega_{1}=\sqrt{\omega_{1}^{2}-a^{2} / 4}, \tilde{\omega}_{2}=\sqrt{\omega_{2}^{2}-a^{2} / 4}$ and

$$
\begin{aligned}
& f_{1}^{1}=f^{1} \frac{\omega_{1}^{2}-\omega^{2}}{\left(\omega_{1}^{2}-\omega^{2}\right)^{2}+a^{2} \omega^{2}}, \\
& f_{2}^{1}=f^{1} \frac{a \omega}{\left(\omega_{1}^{2}-\omega^{2}\right)^{2}+a^{2} \omega^{2}}, \\
& f_{1}^{2}=f^{2} \frac{\omega_{2}^{2}-\omega^{2}}{\left(\omega_{2}^{2}-\omega^{2}\right)^{2}+a^{2} \omega^{2}}, \\
& f_{2}^{2}=f^{2} \frac{a \omega}{\left(\omega_{2}^{2}-\omega^{2}\right)^{2}+a^{2} \omega^{2}} .
\end{aligned}
$$

Moreover, if $\eta_{1}=a / 2 \tilde{\omega}_{1}$ and $\eta_{2}=a / 2 \tilde{\omega}_{2}$, the solution is given in the initial co-ordinate system by

$$
\begin{aligned}
& x=x_{1}, \\
& y=v_{2} x_{1}+x_{2} .
\end{aligned}
$$

3.1.1.2. Gluing at impact times. An impact occurs when $x(t)=x_{\max }$, which is equivalent to $x_{1}(t)=x_{\max }$. If it is assumed that there is an impact at $t_{k}$, on $\left[t_{k-1}, t_{k}\right]$, the solution (16) is given by

$$
\begin{aligned}
& x_{1}(t)=\mathrm{e}^{-a t / 2}\left[A_{k}^{1} \cos \left(\tilde{\omega}_{1} t\right)+B_{k}^{1} \sin \left(\tilde{\omega}_{1} t\right)\right]+f_{1}^{1} \cos (\omega t)+f_{2}^{1} \sin (\omega t), \\
& x_{2}(t)=\mathrm{e}^{-a t / 2}\left[A_{k}^{2} \cos \left(\tilde{\omega}_{2} t\right)+B_{k}^{2} \sin \left(\tilde{\omega}_{2} t\right)\right]+f_{1}^{2} \cos (\omega t)+f_{2}^{2} \sin (\omega t) .
\end{aligned}
$$

From this equation it can be inferred that $t_{k}$ is solution of the equation

$$
f\left(t_{k}\right)=\mathrm{e}^{-a t_{k} / 2}\left[A_{k}^{1} \cos \left(\tilde{\omega}_{1} t_{k}\right)+B_{k}^{1} \sin \left(\tilde{\omega}_{1} t_{k}\right)\right]+f_{1}^{1} \cos \left(\omega t_{k}\right)+f_{2}^{1} \sin \left(\omega t_{k}\right)-x_{\max }=0 .
$$

Moreover, by assumptions on $x$ and $y$, at impact time

$$
\begin{aligned}
& x\left(t_{k}^{+}\right)=x\left(t_{k}^{-}\right), \\
& \dot{x}\left(t_{k}^{+}\right)=-e \dot{x}\left(t_{k}^{-}\right), \\
& y\left(t_{k}^{+}\right)=y\left(t_{k}^{-}\right), \\
& \dot{y}\left(t_{k}^{+}\right)=\dot{y}\left(t_{k}^{-}\right),
\end{aligned}
$$

which yields for the new variables:

$$
\begin{aligned}
& x_{1}\left(t_{k}^{+}\right)=x_{1}\left(t_{k}^{-}\right) \\
& \dot{x}_{1}\left(t_{k}^{+}\right)=-e \dot{x}_{1}\left(t_{k}^{-}\right) \\
& x_{2}\left(t_{k}^{+}\right)=x_{2}\left(t_{k}^{-}\right) \\
& \dot{x}_{2}\left(t_{k}^{+}\right)=\dot{x}_{2}\left(t_{k}^{-}\right)+(1+e) v_{2} \dot{x}_{1}\left(t_{k}^{-}\right) .
\end{aligned}
$$


Thus, obtain the following relationships provide the constants of integration

$$
\begin{aligned}
& A_{k+1}^{1}=A_{k}^{1}-(1+e)_{2} \sin \left(\tilde{\omega}_{1} t_{k}\right) u\left(A_{k}^{1}, B_{k}^{1}, t_{k}\right), \\
& B_{k+1}^{1}=B_{k}^{1}+(1+e) \cos \left(\tilde{\omega}_{1} t_{k}\right) u\left(A_{k}^{1}, B_{k}^{1}, t_{k}\right), \\
& A_{k+1}^{2}=A_{k}^{2}+(1+e) v_{2} \frac{\tilde{\omega}_{1}}{\tilde{\omega}_{2}} \sin \left(\tilde{\omega}_{2} t_{k}\right) u\left(A_{k}^{1}, B_{k}^{1}, t_{k}\right), \\
& B_{k+1}^{2}=B_{k}^{2}-(1+e) v_{2} \frac{\tilde{\omega}_{1}}{\tilde{\omega}_{2}} \cos \left(\tilde{\omega}_{2} t_{k}\right) u\left(A_{k}^{1}, B_{k}^{1}, t_{k}\right),
\end{aligned}
$$

where $u$ is given by

$$
\begin{aligned}
u(A, B, t)= & A\left[\sin \left(\tilde{\omega}_{1} t\right)+\eta_{1} \cos \left(\tilde{\omega}_{1} t\right)\right]+B\left[-\cos \left(\tilde{\omega}_{1} t\right)+\eta_{1} \sin \left(\tilde{\omega}_{1} t\right)\right] \\
& +\mathrm{e}^{a t / 2} \frac{\omega}{\tilde{\omega}_{1}}\left[f_{1}^{1} \sin (\omega t)-f_{2}^{1} \cos (\omega t)\right] .
\end{aligned}
$$

\subsubsection{Search for periodic solutions}

The search for periodic solutions $(x, y)$ is equivalent to the search for periodic solutions $\left(x_{1}, x_{2}\right)$. According to equation (17), $x_{1}=x$, therefore the search for periodic solutions for $x_{1}$ is similar to the one carried out in the case of a single-d.o.f. system. Thus, it is possible to determine $x_{1}^{0}$ and $\dot{x}_{1}^{0}$ so that $x_{1}$ is $(n, k)$-periodic where $k \in\{0,1,2\}$. As regards $x_{2}$, the method is identical; only the recursion that gives $A_{2}^{2}$ and $B_{2}^{2}$ being different. Thus $2 \times 2$ linear systems are obtained in $\left(A_{1}^{2}\left(t_{1}\right), B_{1}^{2}\left(t_{1}\right)\right)$. When the determinant is non-zero, $x_{2}^{0}$ and $\dot{x}_{2}^{0}$ are obtained so that $x_{2}$ is $(n, k)$-periodic where $k \in\{0,1,2\}$.

The initial conditions for the original system co-ordinates $(x, y)$ are given by equation (17):

$$
\begin{aligned}
& x_{0}=x_{1}^{0}, \\
& y_{0}=v_{2} x_{1}^{0}+x_{2}^{0}, \\
& \dot{x}_{0}=\dot{x}_{1}^{0}, \\
& \dot{y}_{0}=v_{2} \dot{x}_{1}^{0}+\dot{x}_{2}^{0} .
\end{aligned}
$$

\subsubsection{Modal superposition}

3.1.3.1. Free oscillations of the system. It was seen in Section 2.3.1 that, in the case of a single-d.o.f. system without external forcing, the number of impacts is finite and the steady state response is periodic with frequency $\omega_{1}$. Hence, in the case of weak coupling, the free response also exhibits a finite number of impacts, and the steady state response for $x_{1}$ is periodic with frequency $\omega_{1}$. As for $x_{2}$, since there are no more impacts once the steady state response is reached, the equation of its movement is given by equation (15):

$$
\ddot{x}_{2}+a \dot{x}_{2}+\omega_{2}^{2} x_{2}=0 .
$$

This is the equation of a classical damped oscillator without external forcing, whose steady state response has frequency $\omega_{2}$. In order to establish a modal superposition formula, it is necessary to start from the natural frequencies $\omega_{1}$ and $\omega_{2}$. Moreover, in that case again, the generalized modes correspond to linear modes. 
3.1.3.2. Generalized masses and modal superposition. Consider a $(n, k)$-periodic solution. The Fourier coefficients of the functions $x_{1}$ and $x_{2}$ can be calculated. These are quite similar to the coefficients found for the single-d.o.f. system. Setting

$$
\begin{aligned}
H_{n, k}^{j}(\omega)= & \frac{2 \tilde{\omega}_{1}}{n T} \frac{1}{\omega_{1}^{2}-\frac{j^{2} \omega^{2}}{n^{2}}+\mathrm{i} a \frac{j \omega}{n}} \\
& \left\{B_{1}^{1}+A_{k+1}^{1} \mathrm{e}^{-a n T / 2} \sin \left(n \tilde{\omega}_{1} T\right)-B_{k+1}^{1} \mathrm{e}^{-a n T / 2} \cos \left(n \tilde{\omega}_{1} T\right)\right. \\
& +\left(\eta_{1}+\mathrm{i} \gamma_{j}^{1}\right)\left[A_{1}^{1}-A_{k+1}^{1} \mathrm{e}^{-a n T / 2} \cos \left(n \tilde{\omega}_{1} T\right)-B_{k+1}^{1} \mathrm{e}^{-a n T / 2} \sin \left(n \tilde{\omega}_{1} T\right)\right] \\
& \left.+(1+e) \sum_{m=1}^{k} \mathrm{e}^{-a t_{m} / 2} \exp \left(-\mathrm{i} \frac{j}{n} \omega t_{m}\right) u\left(A_{m}^{1}, B_{m}^{1}, t_{m}\right)\right\} \\
G_{n, k}^{j}(\omega)= & \frac{2 \tilde{\omega}_{2}}{n T} \frac{1}{\omega_{2}^{2}-\frac{j^{2} \omega^{2}}{n^{2}}+\mathrm{i} a \frac{j \omega}{n}} \\
& \times\left\{B_{1}^{2}+A_{k+1}^{2} \mathrm{e}^{-a n T / 2} \sin \left(n \tilde{\omega}_{2} T\right)-B_{k+1}^{2} \mathrm{e}^{-a n T / 2} \cos \left(n \tilde{\omega}_{2} T\right)\right. \\
& +\left(\eta_{2}+\mathrm{i} \gamma_{j}^{2}\right)\left[A_{1}^{2}-A_{k+1}^{2} \mathrm{e}^{-a n T / 2} \cos \left(n \tilde{\omega}_{2} T\right)-B_{k+1}^{2} \mathrm{e}^{-a n T / 2} \sin \left(n \tilde{\omega}_{2} T\right)\right] \\
& \left.-(1+e) v_{2} \frac{\tilde{\omega}_{1}}{\tilde{\omega}_{2}} \sum_{m=1}^{k} \mathrm{e}^{-a t_{m} / 2} \exp \left(-\mathrm{i} \frac{j}{n} \omega t_{m}\right) u\left(A_{m}^{1}, B_{m}^{1}, t_{m}\right)\right\}
\end{aligned}
$$

with $\gamma_{j}^{1}=j \omega / n \tilde{\omega}_{1}$ and $\gamma_{j}^{2}=j \omega / n \tilde{\omega}_{2}$, the Fourier coefficients are given by

$$
\begin{aligned}
& c_{j}^{1}(\omega)=\frac{f_{1}^{1}-\mathrm{i} f_{2}^{1}}{2} \delta_{j}^{n}+\frac{f_{1}^{1}+\mathrm{i} f_{2}^{1}}{2} \delta_{j}^{-n}+\frac{H_{n, k}^{j}(\omega)}{2}, \\
& c_{j}^{2}(\omega)=\frac{f_{1}^{2}-\mathrm{i} f_{2}^{2}}{2} \delta_{j}^{n}+\frac{f_{1}^{2}+\mathrm{i} f_{2}^{2}}{2} \delta_{j}^{-n}+\frac{G_{n, k}^{j}(\omega)}{2} .
\end{aligned}
$$

The Fourier coefficient corresponding to the $n$th harmonic are inferred by using the coordinate transformation (17)

$$
\begin{aligned}
& c_{n}^{x}(\omega)=c_{n}^{1}(\omega)=\frac{f^{1}}{2 m_{1}^{n, k} \Delta I_{1}}, \\
& c_{n}^{y}(\omega)=v_{2} c_{n}^{1}(\omega)+c_{n}^{2}(\omega)=v_{2} \frac{f^{1}}{2 m_{1}^{n, k} \Delta I_{1}}+\frac{f^{2}}{2 m_{2}^{n, k} \Delta I_{2}},
\end{aligned}
$$

where, $\Delta I_{1}=\omega_{1}^{2}-\omega^{2}+a i \omega, \Delta I_{2}=\omega_{2}^{2}-\omega^{2}+a i \omega$ and

$$
\begin{aligned}
& m_{1}^{n, k}=\frac{1}{1+H_{n, k}^{n}(\omega) / f^{1}}, \\
& m_{2}^{n, k}=\frac{1}{1+G_{n, k}^{n}(\omega) / f^{2}} .
\end{aligned}
$$


The $n$th Fourier coefficients are then in the original basis:

$$
\begin{aligned}
& c_{n}^{x}(\omega)=\frac{f_{1}}{2 m_{1}^{n, k} \Delta I_{1}}, \\
& c_{n}^{y}(\omega)=v_{2} \frac{f_{1}}{2 m_{1}^{n, k} \Delta I_{1}}+\frac{-v_{2} f_{1}+f_{2}}{2 m_{2}^{n, k} \Delta I_{2}} .
\end{aligned}
$$

The contribution of the $n$th harmonic to the Fourier spectrum is given (as seen in equation (12)) by

$$
\begin{aligned}
& \mathscr{A}_{n}^{X}(\omega)=\left|X_{n}(\omega)\right|=\left|\frac{f_{1}}{m_{1}^{n, k} \Delta I_{1}}\right|, \\
& \mathscr{A}_{n}^{Y}(\omega)=\left|Y_{n}(\omega)\right|=\left|v_{2} \frac{f_{1}}{m_{1}^{n, k} \Delta I_{1}}+\frac{-v_{2} f_{1}+f_{2}}{m_{2}^{n, k} \Delta I_{2}}\right|,
\end{aligned}
$$

which can also be written as

$$
\left(\begin{array}{l}
X_{n}(\omega) \\
Y_{n}(\omega)
\end{array}\right)=\frac{\left(\begin{array}{cc}
1 & 0 \\
v_{2} & 0
\end{array}\right)}{m_{1}^{n, k} \Delta I_{1}}\left(\begin{array}{l}
f_{1} \\
f_{2}
\end{array}\right)+\frac{\left(\begin{array}{cc}
0 & 0 \\
-v_{2} & 1
\end{array}\right)}{m_{2}^{n, k} \Delta I_{2}}\left(\begin{array}{l}
f_{1} \\
f_{2}
\end{array}\right) .
$$

If

$$
\mathbf{A}_{1}=\left(\begin{array}{ll}
1 & 0 \\
v_{2} & 0
\end{array}\right) \quad \text { and } \quad \mathbf{A}_{2}=\left(\begin{array}{rr}
0 & 0 \\
-v_{2} & 1
\end{array}\right)
$$

we can express $\mathbf{A}_{1}$ in the form $\mathbf{T}_{1}^{t} \mathbf{T}_{1}^{\prime}$, and $\mathbf{A}_{2}$ in the form $\mathbf{T}_{2}^{t} \mathbf{T}_{2}^{\prime}$ where $\mathbf{T}_{1}, \mathbf{T}_{1}^{\prime}, \mathbf{T}_{2}$ and $\mathbf{T}_{2}^{\prime}$ denote the generalized modes of the system.

If $\mathbf{T}_{1}=\left(a_{1}, b_{1}\right)$ and $\mathbf{T}_{1}^{\prime}=\left(a_{1}^{\prime}, b_{1}^{\prime}\right)$ : then

$$
\mathbf{T}_{1}^{t} \mathbf{T}_{1}^{\prime}=\left(\begin{array}{ll}
a_{1} a_{1}^{\prime} & a_{1} b_{1}^{\prime} \\
b_{1} a_{1}^{\prime} & b_{1} b_{1}^{\prime}
\end{array}\right),
$$

three equations with four unknowns are obtained

$$
\begin{aligned}
& b_{1}^{\prime}=0, \\
& a_{1} a_{1}^{\prime}=1, \\
& b_{1} a_{1}^{\prime}=v_{2} .
\end{aligned}
$$

It is thus possible to arbitrarily set one of the unknowns; for example, as in the case of linear modes, if $a_{1}^{\prime}=1$, then

$$
\begin{aligned}
& a_{1}=1, \\
& b_{1}=v_{2},
\end{aligned}
$$

hence

$$
\begin{aligned}
& \mathbf{T}_{1}=\left(1, v_{2}\right), \\
& \mathbf{T}_{1}^{\prime}=(1,0) .
\end{aligned}
$$


The same type of calculations and assumptions for $A_{2}$ lead to

$$
\begin{aligned}
& \mathbf{T}_{2}=(0,1), \\
& \mathbf{T}_{2}^{\prime}=\left(-v_{2}, 1\right) .
\end{aligned}
$$

Using equations (23) and (24), relation (22) can then be written in the form

$$
\left(\begin{array}{c}
X_{n}(\omega) \\
Y_{n}(\omega)
\end{array}\right)=\frac{\mathbf{T}_{1}^{t} \mathbf{T}_{1}^{\prime}}{m_{1}^{n, k} \Delta I_{1}(\omega)}\left(\begin{array}{c}
f_{1} \\
f_{2}
\end{array}\right)+\frac{\mathbf{T}_{2}^{t} \mathbf{T}_{2}^{\prime}}{m_{2}^{n, k} \Delta I_{2}(\omega)}\left(\begin{array}{c}
f_{1} \\
f_{2}
\end{array}\right) .
$$

This has just established a modal superposition formula holding for a two-d.o.f. system with weak coupling. This formula links the $n$th harmonic amplitude to the free response and the forcing by means of a modal mass. The vectors $\mathbf{T}_{1}, \mathbf{T}_{1}^{\prime}, \mathbf{T}_{2}$ and $\mathbf{T}_{2}^{\prime}$ represent the modes (left and right respectively). Note that $\mathbf{T}_{1}$ and $\mathbf{T}_{2}$ are merely the eigenvectors associated with $\omega_{1}^{2}$ and $\omega_{2}^{2}$.

Finally, as in the case of the single-d.o.f. system, there is no unique modal superposition formula which holds in all cases, but according to the period of the response and the number of impacts per cycle, it is necessary to choose the right formula.

Remark. The modal superposition formula seems to exhibit a reciprocity breaking compared to the linear case: $T_{1}$ and $T_{1}^{\prime}$ are different whatever choice is made when setting one of the unknowns. Indeed, the second component of $T_{1}^{\prime}$ is always zero whereas that of $T_{1}$ is always non-zero. This is in fact due to the choice of the model, for which $k_{1}=0$ and $k_{2} \neq 0$.

3.1.3.3. Examples of modal superposition. For the first example, the following parameters are chosen: $\omega_{1}=2 \cdot 5, \omega_{2}=3 \cdot 8, a=0 \cdot 05, e=0 \cdot 9, x_{\max }=14, f_{1}=20, f_{2}=18$ and $k_{2}=1$.

The second example is derived from the single-d.o.f. case and the chosen parameters are the following: $\omega_{1}=1, \omega_{2}=3 \cdot 8, a=0 \cdot 02, e=0 \cdot 9, x_{\max }=1, f_{1}=20, f_{2}=18$ and $k_{2}=1$. The spectral amplitude for $x$ is the same one as that obtained in the single-d.o.f. case. The continuous d.o.f. $y$ exhibits a resonance for $\omega \simeq \omega_{2}$ similar to the linear case, but several additional secondary resonances occur for $\omega \simeq \omega_{2} / 2$ and $\omega \simeq \omega_{2} / 3$. Furthermore, for the frequency associated with a spectral amplitude peak for $x$, a small peak for $y$ is found, which can become large if $k_{2}$ is sufficiently large. Lastly, if $\omega_{1}$ is close to $\omega_{2} / 2$ and $k_{2}$ is rather large, then the main peak of resonance can occur in the neighbourhood of $\omega_{2} / 2$.

\subsection{STRONG COUPLING}

In this section, the system (14) with $k_{1} \neq 0$ is studied.

\subsubsection{Analytical solution of the system}

3.2.1.1. Decoupling equations. System (14) will be decoupled in order to be able to write the solutions $x$ and $y$ explicitly. The matrix $K$ has the following characteristic polynomial:

$$
P_{K}(\lambda)=\lambda^{2}-\left(\omega_{1}^{2}+\omega_{2}^{2}\right) \lambda+\omega_{1}^{2} \omega_{2}^{2}-k_{1} k_{2}
$$

with discriminant $\Delta=\left(\omega_{1}^{2}-\omega_{2}^{2}\right)^{2}+4 k_{1} k_{2}$. 

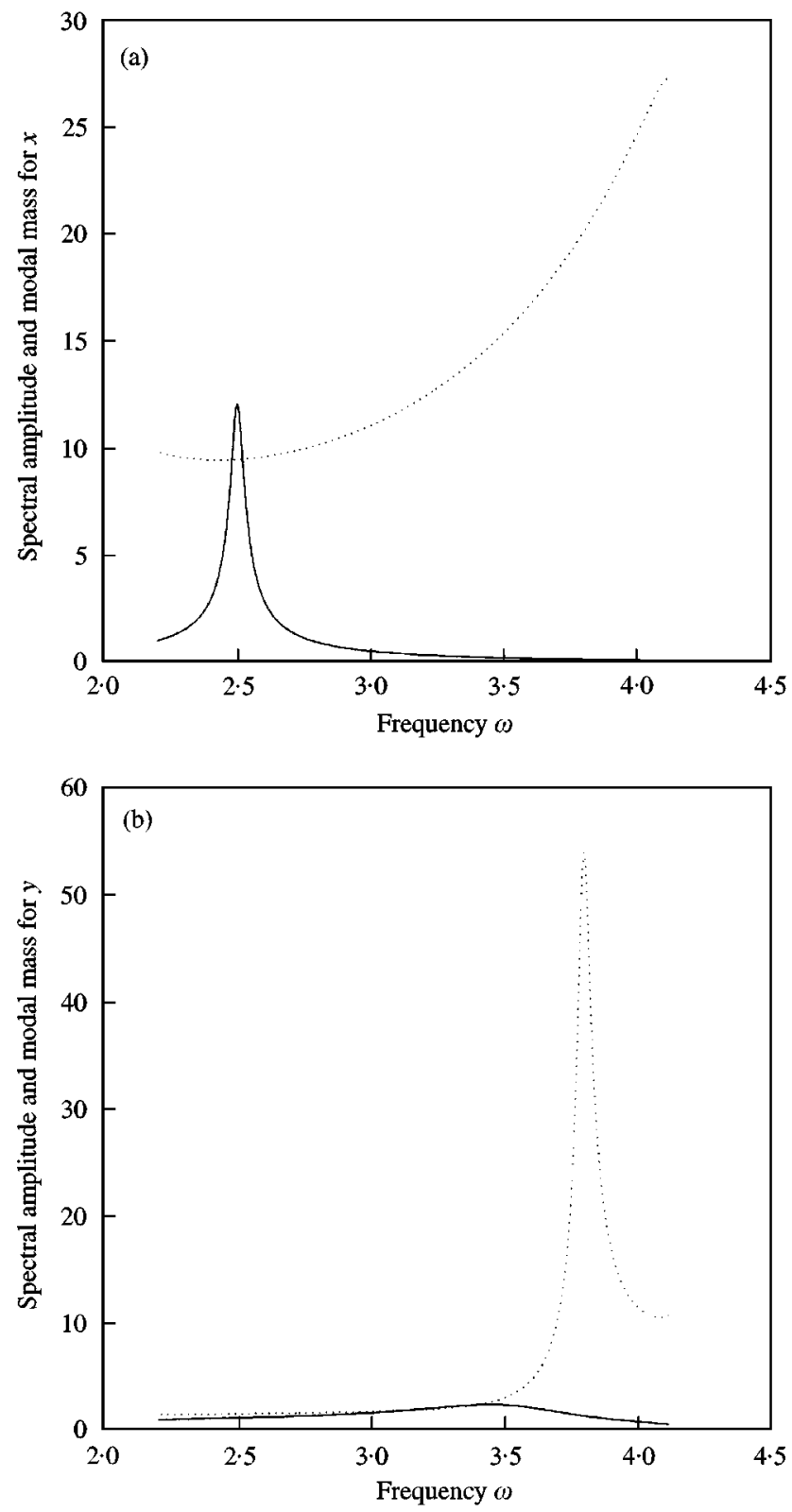

Figure 19. First-harmonic amplitude (dotted curve) and modal mass (solid curve) of the (1,1)-periodic responses: (a) First degree of freedom $x$; (b) second degree of freedom $y$.

The system parameters will be chosen so that $\Delta>0$. Then the matrix $K$ admits two distinct real eigenvalues:

$$
\begin{aligned}
& \lambda_{1}=\frac{\omega_{1}^{2}+\omega_{2}^{2}-\sqrt{\Delta}}{2}, \\
& \lambda_{2}=\frac{\omega_{1}^{2}+\omega_{2}^{2}+\sqrt{\Delta}}{2} .
\end{aligned}
$$



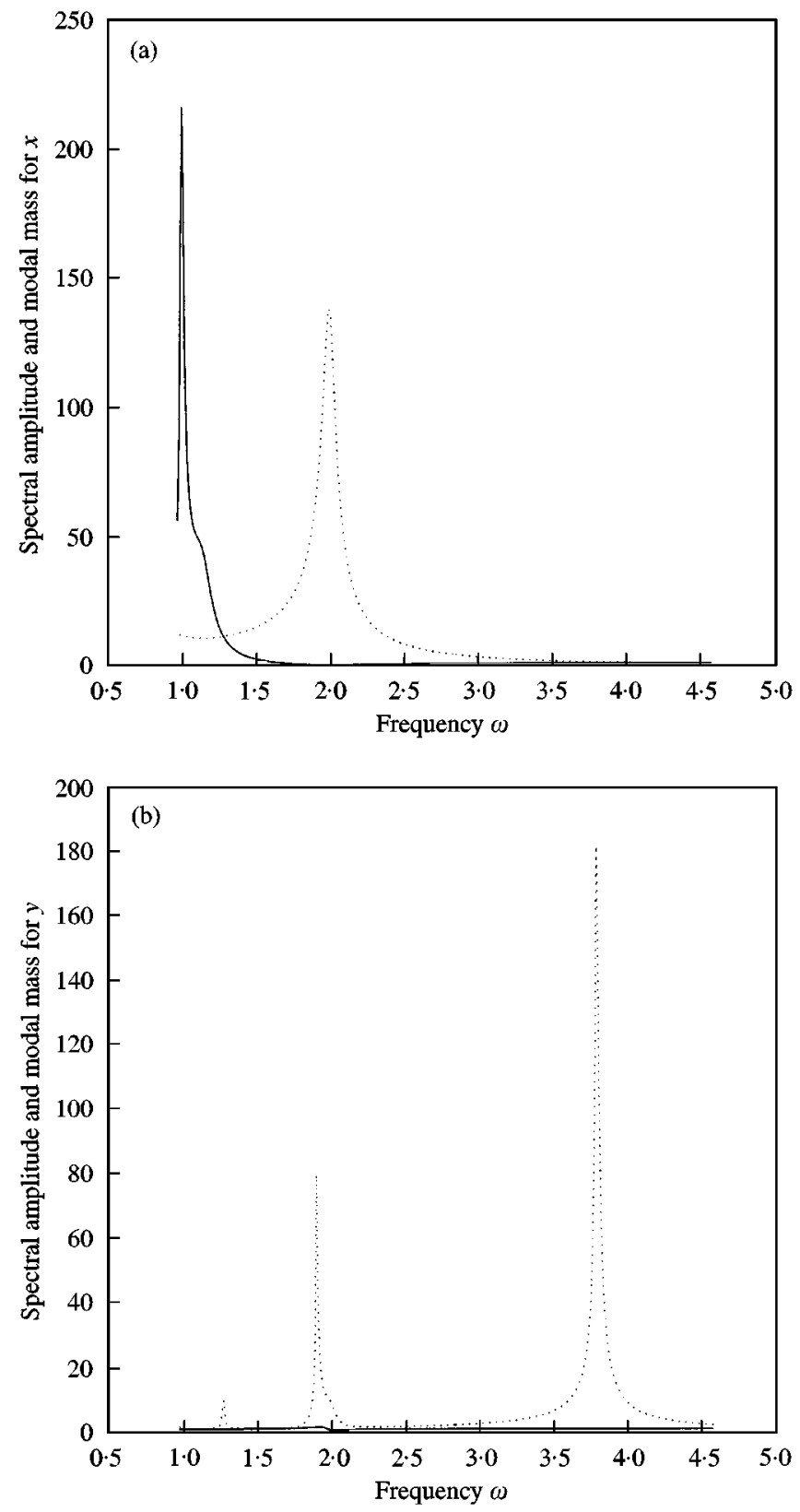

Figure 20. First-harmonic amplitude (dotted curve) and modal mass (solid curve) of the (1,1)-periodic responses: (a) First degree of freedom $x$; (b) second degree of freedom $y$.

Assume that $\omega_{1} \neq \omega_{2}$; if $v_{1}=k_{1} /\left(\omega_{1}^{2}-\omega_{2}^{2}\right)$ and $v_{2}=k_{2} /\left(\omega_{1}^{2}-\omega_{2}^{2}\right)$, then two eigenvectors associated with $\lambda_{1}$ and $\lambda_{2}$ are respectively $\left(\begin{array}{l}1 \\ v_{2}\end{array}\right)$ and $\left(\begin{array}{c}v_{1} \\ -1\end{array}\right)$, which define the matrix

$$
\mathbf{P}=\left(\begin{array}{cc}
1 & -v_{1} \\
v_{2} & 1
\end{array}\right) .
$$


The assumption $\Delta>0$ implies that its determinant is nonzero, and $\mathbf{P}$ is invertible with

$$
\mathbf{P}^{-1}=\frac{1}{1+v_{1} v_{2}}\left(\begin{array}{cc}
1 & v_{1} \\
-v_{2} & 1
\end{array}\right) .
$$

Then, if $\mathbf{X}=\mathbf{P Y}$, the system (14) is multiplied by $\mathbf{P}^{-1}$ in order to obtain

$$
\ddot{\mathbf{Y}}+a \dot{\mathbf{Y}}+\mathbf{P}^{-1} \mathbf{K P Y}=\mathbf{P}^{-1} \mathbf{F} \cos (\omega t)+\text { impact }
$$

Let

$$
\begin{aligned}
& f^{1}=\frac{f_{1}+v_{1} f_{2}}{1+v_{1} v_{2}}, \\
& f^{2}=\frac{f_{2}-v_{2} f_{1}}{1+v_{1} v_{2}} .
\end{aligned}
$$

The new system obtained using the new basis is given by

$$
\begin{aligned}
& \ddot{x}_{1}+a \dot{x}_{1}+\lambda_{1} x_{1}=f^{1} \cos (\omega t), \\
& \ddot{x}_{2}+a \dot{x}_{2}+\lambda_{2} x_{2}=f^{2} \cos (\omega t)
\end{aligned}
$$

as long as there is no impact. Thus these equations can be solved easily;

$$
\begin{aligned}
& x_{1}(t)=\mathrm{e}^{-a t / 2}\left[A^{1} \cos \left(\tilde{\omega}_{1} t\right)+B^{1} \sin \left(\tilde{\omega}_{1} t\right)\right]+f_{1}^{1} \cos (\omega t)+f_{2}^{1} \sin (\omega t), \\
& x_{2}(t)=\mathrm{e}^{-a t / 2}\left[A^{2} \cos \left(\tilde{\omega}_{2} t\right)+B^{2} \sin \left(\tilde{\omega}_{2} t\right)\right]+f_{1}^{2} \cos (\omega t)+f_{2}^{2} \sin (\omega t)
\end{aligned}
$$

with $\tilde{\omega}_{1}=\sqrt{\lambda_{1}-a^{2} / 4}, \tilde{\omega}_{2}=\sqrt{\lambda_{2}-a^{2} / 4}$ and

$$
\begin{aligned}
& f_{1}^{1}=f^{1} \frac{\lambda_{1}-\omega^{2}}{\left(\lambda_{1}-\omega^{2}\right)^{2}+a^{2} \omega^{2}}, \\
& f_{2}^{1}=f^{1} \frac{a \omega}{\left(\lambda_{1}-\omega^{2}\right)^{2}+a^{2} \omega^{2}}, \\
& f_{1}^{2}=f^{2} \frac{\lambda_{2}-\omega^{2}}{\left(\lambda_{2}-\omega^{2}\right)^{2}+a^{2} \omega^{2}}, \\
& f_{2}^{2}=f^{2} \frac{a \omega}{\left(\lambda_{2}-\omega^{2}\right)^{2}+a^{2} \omega^{2}} .
\end{aligned}
$$

The solution in the original basis is finally given by

$$
\begin{aligned}
& x=x_{1}-v_{1} x_{2}, \\
& y=v_{2} x_{1}+x_{2} .
\end{aligned}
$$

3.2.1.2. Gluing solutions at impact times. An impact occurs when $x(t)=x_{\text {max }}$, namely when $x_{1}(t)-v_{1} x_{2}(t)=x_{\max }$. Assume that there is an impact at $t_{k}$. For $t \in\left[t_{k-1}, t_{k}\right]$, the decoupled solution is given by

$$
\begin{aligned}
& x_{1}(t)=\mathrm{e}^{-a t / 2}\left[A_{k}^{1} \cos \left(\tilde{\omega}_{1} t\right)+B_{k}^{1} \sin \left(\tilde{\omega}_{1} t\right)\right]+f_{1}^{1} \cos (\omega t)+f_{2}^{1} \sin (\omega t), \\
& x_{2}(t)=\mathrm{e}^{-a t / 2}\left[A_{k}^{2} \cos \left(\tilde{\omega}_{2} t\right)+B_{k}^{2} \sin \left(\tilde{\omega}_{2} t\right)\right]+f_{1}^{2} \cos (\omega t)+f_{2}^{2} \sin (\omega t) .
\end{aligned}
$$


From these equations it can be inferred that the equation verified by $t_{k}$ is

$$
\begin{aligned}
f\left(t_{k}\right)= & \mathrm{e}^{-a t_{k} / 2}\left\{A_{k}^{1} \cos \left(\tilde{\omega}_{1} t_{k}\right)+B_{k}^{1} \sin \left(\tilde{\omega}_{1} t_{k}\right)-v_{1}\left[A_{k}^{2} \cos \left(\tilde{\omega}_{2} t_{k}\right)+B_{k}^{2} \sin \left(\tilde{\omega}_{2} t_{k}\right)\right]\right\} \\
& +\left(f_{1}^{1}-v_{1} f_{1}^{2}\right) \cos \left(\omega t_{k}\right)+\left(f_{2}^{1}-v_{1} f_{2}^{2}\right) \sin \left(\omega t_{k}\right)-x_{\max }=0 .
\end{aligned}
$$

Yet by assumptions on $x$ and $y$, at the impact time:

$$
\begin{aligned}
& x\left(t_{k}^{+}\right)=x\left(t_{k}^{-}\right), \\
& \dot{x}\left(t_{k}^{+}\right)=-e \dot{x}\left(t_{k}^{-}\right), \\
& y\left(t_{k}^{+}\right)=y\left(t_{k}^{-}\right), \\
& \dot{y}\left(t_{k}^{+}\right)=\dot{y}\left(t_{k}^{-}\right)
\end{aligned}
$$

which yields, according to (28),

$$
\begin{aligned}
& x_{1}\left(t_{k}^{+}\right)=x_{1}\left(t_{k}^{-}\right) \\
& \dot{x}_{1}\left(t_{k}^{+}\right)=\left(1-\frac{1+e}{1+v_{1} v_{2}}\right) \dot{x}_{1}\left(t_{k}^{-}\right)+\frac{(1+e) v_{1}}{1+v_{1} v_{2}} \dot{x}_{2}\left(t_{k}^{-}\right), \\
& x_{2}\left(t_{k}^{+}\right)=x_{2}\left(t_{k}^{-}\right) \\
& \dot{x}_{2}\left(t_{k}^{+}\right)=\frac{(1+e) v_{2}}{1+v_{1} v_{2}} \dot{x}_{1}\left(t_{k}^{-}\right)+\left(\frac{1+e}{1+v_{1} v_{2}}-e\right) \dot{x}_{2}\left(t_{k}^{-}\right) .
\end{aligned}
$$

For $i \in\{1,2\}$,

$$
\begin{aligned}
u_{i}(k) & \left.=A_{k}^{i} \sin \left(\tilde{\omega}_{i} t_{k}\right)+\eta_{i} \cos \left(\tilde{\omega}_{i} t_{k}\right)\right]+B_{k}^{i}\left[-\cos \left(\tilde{\omega}_{i} t_{k}\right)+\eta_{i} \sin \left(\tilde{\omega}_{i} t_{k}\right)\right] \\
& +\frac{\omega}{\tilde{\omega}_{i}} \mathrm{e}^{a t_{k} / 2}\left[f_{1}^{i} \sin \left(\omega t_{k}\right)-f_{2}^{i} \cos \left(\omega t_{k}\right)\right],
\end{aligned}
$$

then:

$$
\begin{aligned}
& A_{k+1}^{1}=A_{k}^{1}-\frac{1+e}{1+v_{1} v_{2}} \sin \left(\tilde{\omega}_{1} t_{k}\right)\left[u_{1}(k)-v_{1} \frac{\tilde{\omega}_{2}}{\tilde{\omega}_{1}} u_{2}(k)\right], \\
& B_{k+1}^{1}=B_{k}^{1}+\frac{1+e}{1+v_{1} v_{2}} \cos \left(\tilde{\omega}_{1} t_{k}\right)\left[u_{1}(k)-v_{1} \frac{\tilde{\omega}_{2}}{\tilde{\omega}_{1}} u_{2}(k)\right], \\
& A_{k+1}^{2}=A_{k}^{2}+\frac{(1+e) v_{2}}{1+v_{1} v_{2}} \sin \left(\tilde{\omega}_{2} t_{k}\right)\left[\frac{\tilde{\omega}_{1}}{\tilde{\omega}_{2}} u_{1}(k)-v_{1} u_{2}(k)\right], \\
& B_{k+1}^{2}=B_{k}^{2}-\frac{(1+e) v_{2}}{1+v_{1} v_{2}} \cos \left(\tilde{\omega}_{2} t_{k}\right)\left[\frac{\tilde{\omega}_{1}}{\tilde{\omega}_{2}} u_{1}(k)-v_{1} u_{2}(k)\right] .
\end{aligned}
$$

\subsubsection{Search for periodic solutions}

The search for periodic solutions $(x, y)$ is equivalent to the search for periodic solutions $\left(x_{1}, x_{2}\right)$. Only $(n, 0)$ and $(n, 1)$-periodic solutions will be sought. The following calculations are similar to those found in reference [76], with the addition of damping. 
3.2.2.1. (n,0)-periodic solutions. As for the single d.o.f system, it is easy to prove that the system admits a $(n, 0)$-periodic solution if, and only if, $x_{1}$ and $x_{2}$ are given by

$$
\begin{aligned}
& x_{1}(t)=f_{1}^{1} \cos (\omega t)+f_{2}^{1} \sin (\omega t), \\
& x_{2}(t)=f_{1}^{2} \cos (\omega t)+f_{2}^{2} \sin (\omega t) .
\end{aligned}
$$

According to equation (28):

$$
x(t)=\left(f_{1}^{1}-v_{1} f_{1}^{2}\right) \cos (\omega t)+\left(f_{2}^{1}-v_{1} f_{2}^{2}\right) \sin (\omega t) .
$$

It is thus possible to get results similar to those obtained for a single-d.o.f. system in section 2.2.1. This gives a condition that a $(n, 0)$-periodic solution exists; it requires $\left(f_{1}^{1}-v_{1} f_{1}^{2}\right)^{2}+\left(f_{2}^{1}-v_{1} f_{2}^{2}\right)^{2} \leqslant x_{\max }^{2}$. From this inequality, a fourth degree polynomial in $\omega^{2}$ can be obtained, allowing the values of $\omega$ for which the system admits a (n,0)-periodic response to be determined. The remark made in Section 2.2.1 still holds; the existence of $(n, 0)$-periodic solutions requires $x_{\max }>0$.

3.2.2.2. (n,1)-periodic solutions. $n T$-periodicity requires

$$
\begin{aligned}
& x_{1}(n T)=x_{1}^{0}, \\
& \dot{x}_{1}(n T)=\dot{x}_{1}^{0}, \\
& x_{2}(n T)=x_{2}^{0}, \\
& \dot{x}_{2}(n T)=\dot{x}_{2}^{0}
\end{aligned}
$$

or using linear combination, for a solution with one impact per cycle:

$$
\begin{aligned}
& A_{2}^{1} \cos \left(n \tilde{\omega}_{1} T\right)+B_{2}^{1} \sin \left(n \tilde{\omega}_{1} T\right)-\mathrm{e}^{a n T / 2} A_{1}^{1}=0 \\
& A_{2}^{2} \cos \left(n \tilde{\omega}_{2} T\right)+B_{2}^{2} \sin \left(n \tilde{\omega}_{2} T\right)-\mathrm{e}^{a n T / 2} A_{1}^{2}=0 \\
& A_{2}^{1} \sin \left(n \tilde{\omega}_{1} T\right)-B_{2}^{1} \cos \left(n \tilde{\omega}_{1} T\right)+\mathrm{e}^{a n T / 2} B_{1}^{1}=0 \\
& A_{2}^{2} \sin \left(n \tilde{\omega}_{2} T\right)+B_{2}^{2} \cos \left(n \tilde{\omega}_{2} T\right)-\mathrm{e}^{a n T / 2} B_{1}^{2}=0 .
\end{aligned}
$$

Using equation (29), a $4 \times 4$ linear system in $\left(A_{1}^{1}, B_{1}^{1}, A_{1}^{2}, B_{1}^{2}\right)$ is obtained. The resolution of this system leads to $A_{1}^{1}\left(t_{1}\right), B_{1}^{1}\left(t_{1}\right), A_{1}^{2}\left(t_{1}\right)$ and $B_{1}^{2}\left(t_{1}\right)$.

It remains to determine $t_{1}$ via the equation

$$
\begin{aligned}
f\left(t_{1}\right)= & \mathrm{e}^{-a t_{1} / 2}\left\{A_{1}^{1}\left(t_{1}\right) \cos \left(\tilde{\omega}_{1} t_{1}\right)+B_{1}^{1}\left(t_{1}\right) \sin \left(\tilde{\omega}_{1} t_{1}\right)\right. \\
& \left.-v_{1}\left[A_{1}^{2}\left(t_{1}\right) \cos \left(\tilde{\omega}_{2} t_{1}\right)+B_{1}^{2}\left(t_{1}\right) \sin \left(\tilde{\omega}_{2} t_{1}\right)\right]\right\} \\
& +\left(f_{1}^{1}-v_{1} f_{1}^{2}\right) \cos \left(\omega t_{1}\right)+\left(f_{2}^{1}-v_{1} f_{2}^{2}\right) \sin \left(\omega t_{1}\right)-x_{\max }=0 .
\end{aligned}
$$

Initial conditions of the system leading to a $(n, 1)$-periodic solution are then given by

$$
\begin{aligned}
& x_{0}=A_{1}^{1}\left(t_{1}\right)+f_{1}^{1}-v_{1}\left(A_{1}^{2}\left(t_{1}\right)+f_{1}^{2}\right), \\
& y_{0}=v_{2}\left(A_{1}^{1}\left(t_{1}\right)+f_{1}^{1}\right)+A_{1}^{2}\left(t_{1}\right)+f_{1}^{2},
\end{aligned}
$$




$$
\begin{aligned}
& \dot{x}_{0}=\tilde{\omega}_{1}\left[B_{1}^{1}\left(t_{1}\right)-\eta_{1} A_{1}^{1}\left(t_{1}\right)\right]+f_{2}^{1} \omega-v_{1} \tilde{\omega}_{2}\left[B_{1}^{2}\left(t_{1}\right)-\eta_{2} A_{1}^{2}\left(t_{1}\right)\right]-v_{1} f_{2}^{2} \omega, \\
& \dot{y}_{0}=v_{2} \tilde{\omega}_{1}\left[B_{1}^{1}\left(t_{1}\right)-\eta_{1} A_{1}^{1}\left(t_{1}\right)\right]+v_{2} f_{2}^{1} \omega+\tilde{\omega}_{2}\left[B_{1}^{2}\left(t_{1}\right)-\eta_{2} A_{1}^{2}\left(t_{1}\right)\right]+f_{2}^{2} \omega .
\end{aligned}
$$

\subsubsection{Modal superpositon}

3.2.3.1. Free oscillation of the system. It can be shown that the system (14) without external forcing has a finite number of impacts. Thus the steady state of the system (26) with $f^{1}=f^{2}=0$ is periodic with frequency $\lambda_{1}$ for $x_{1}$ and $\lambda_{2}$ for $x_{2}$ : these two frequencies will be used as natural frequencies for the forced system.

3.2.3.2. Generalized masses and modal superposition. It is now possible to consider a $(n, k)$-periodic solution and to calculate the Fourier coefficients of $x_{1}$ and $x_{2}$. The calculation of $c_{j}^{1}(\omega)$ and $c_{j}^{2}(\omega)$ is similar to the one carried out in the weak coupling case: only the recursive relation (29) changes. In this case

$$
\begin{aligned}
H_{n, k}^{j}(\omega)= & \frac{2 \tilde{\omega}_{1}}{n T} \frac{1}{\omega_{1}^{2}-\frac{j^{2} \omega^{2}}{n^{2}}+\mathrm{i} a \frac{j \omega}{n}} \\
& \times\left\{B_{1}^{1}+A_{k+1}^{1} \mathrm{e}^{-a n T / 2} \sin \left(n \tilde{\omega}_{1} T\right)-B_{k+1}^{1} \mathrm{e}^{-a n T / 2} \cos \left(n \tilde{\omega}_{1} T\right)\right. \\
& +\left(\eta_{1}+\mathrm{i} \gamma_{j}^{1}\right)\left[A_{1}^{1}-A_{k+1}^{1} \mathrm{e}^{-a n T / 2} \cos \left(n \tilde{\omega}_{1} T\right)-B_{k+1}^{1} \mathrm{e}^{-a n T / 2} \sin \left(n \tilde{\omega}_{1} T\right)\right] \\
& \left.+\frac{1+e}{1+v_{1} v_{2}} \sum_{m=1}^{k} \mathrm{e}^{-a t_{m} / 2} \exp \left(-\mathrm{i} \frac{j}{n} \omega t_{m}\right)\left[u_{1}(m)-v_{1} \frac{\tilde{\omega}_{2}}{\tilde{\omega}_{1}} u_{2}(m)\right]\right\} \\
G_{n, k}^{j}(\omega)= & \frac{2 \tilde{\omega}_{2}}{n T} \frac{j^{2} \omega^{2}}{n^{2}}+\mathrm{i} a \frac{j \omega}{n} \\
& \left\{B_{1}^{2}+A_{k+1}^{2} \mathrm{e}^{-a n T / 2} \sin \left(n \tilde{\omega}_{2} T\right)-B_{k+1}^{2} \mathrm{e}^{-a n T / 2} \cos \left(n \tilde{\omega}_{2} T\right)\right. \\
& +\left(\eta_{2}+\mathrm{i} \gamma_{j}^{2}\right)\left[A_{1}^{2}-A_{k+1}^{2} \mathrm{e}^{-a n T / 2} \cos \left(n \tilde{\omega}_{2} T\right)-B_{k+1}^{2} \mathrm{e}^{-a n T / 2} \sin \left(n \tilde{\omega}_{2} T\right)\right] \\
& \left.-\frac{(1+e) v_{2}}{1+v_{1} v_{2}} \sum_{m=1}^{k} \mathrm{e}^{-a t_{m} / 2} \exp \left(-\mathrm{i} \frac{j}{n} \omega t_{m}\right)\left[\frac{\tilde{\omega}_{1}}{\tilde{\omega}_{2}} u_{1}(m)-v_{1} u_{2}(m)\right]\right\}
\end{aligned}
$$

with $\gamma_{j}^{1}=j \omega / n \tilde{\omega}_{1}$ and $\gamma_{j}^{2}=j \omega / n \tilde{\omega}_{2}$. Then

$$
\begin{aligned}
& c_{j}^{1}(\omega)=\frac{f_{1}^{1}-\mathrm{i} f_{2}^{1}}{2} \delta_{j}^{n}+\frac{f_{1}^{1}+\mathrm{i} f_{2}^{1}}{2} \delta_{j}^{-n}+\frac{H_{n, k}^{j}(\omega)}{2}, \\
& c_{j}^{2}(\omega)=\frac{f_{1}^{2}-\mathrm{i} f_{2}^{2}}{2} \delta_{j}^{n}+\frac{f_{1}^{2}+\mathrm{i} f_{2}^{2}}{2} \delta_{j}^{-n}+\frac{G_{n, k}^{j}(\omega)}{2} .
\end{aligned}
$$


The $n$th Fourier coefficients of the system by the basis change (28) are then

$$
\begin{aligned}
& c_{n}^{x}(\omega)=c_{n}^{1}(\omega)-v_{1} c_{n}^{2}(\omega)=\frac{f^{1}}{2 m_{1}^{n, k} \Delta I_{1}}-v_{1} \frac{f^{2}}{2 m_{2}^{n, k} \Delta I_{2}}, \\
& c_{n}^{y}(\omega)=v_{2} c_{n}^{1}(\omega)+c_{n}^{2}(\omega)=v_{2} \frac{f^{1}}{2 m_{1}^{n, k} \Delta I_{1}}+\frac{f^{2}}{2 m_{2}^{n, k} \Delta I_{2}},
\end{aligned}
$$

where $\Delta I_{1}=\lambda_{1}-\omega^{2}+a \mathrm{i} \omega, \Delta I_{2}=\lambda_{2}-\omega^{2}+a \mathrm{i} \omega$ and

$$
\begin{aligned}
m_{1}^{n, k} & =\frac{1}{1+H_{n, k}^{n}(\omega) / f^{1}}, \\
m_{2}^{n, k} & =\frac{1}{1+G_{n, k}^{n}(\omega) / f^{2}} .
\end{aligned}
$$

In the original basis,

$$
\begin{aligned}
& c_{n}^{x}(\omega)=\frac{1}{1+v_{1} v_{2}}\left[\frac{f_{1}+v_{1} f_{2}}{2 m_{1}^{n, k} \Delta I_{1}}-v_{1} \frac{f_{2}-v_{2} f_{1}}{2 m_{2}^{n, k} \Delta I_{2}}\right], \\
& c_{n}^{y}(\omega)=\frac{1}{1+v_{1} v_{2}}\left[v_{2} \frac{f_{1}+v_{1} f_{2}}{2 m_{1}^{n, k} \Delta I_{1}}+\frac{f_{2}-v_{2} f_{1}}{2 m_{2}^{n, k} \Delta I_{2}}\right] .
\end{aligned}
$$

The contribution of the $n$th harmonic is (as for the weak coupling in equation (21)) given by

$$
\left(\begin{array}{l}
X_{n}(\omega) \\
Y_{n}(\omega)
\end{array}\right)=\frac{1}{1+v_{1} v_{2}}\left\{\frac{\left(\begin{array}{cc}
1 & v_{1} \\
v_{2} & v_{1} v_{2}
\end{array}\right)}{m_{1}^{n, k} \Delta I_{1}}\left(\begin{array}{l}
f_{1} \\
f_{2}
\end{array}\right)+\frac{\left(\begin{array}{cc}
v_{1} v_{2} & -v_{1} \\
-v_{2} & 1
\end{array}\right)}{m_{2}^{n, k} \Delta I_{2}}\left(\begin{array}{l}
f_{1} \\
f_{2}
\end{array}\right)\right\}
$$

Let

$$
\mathbf{A}_{1}=\frac{1}{1+v_{1} v_{2}}\left(\begin{array}{cc}
1 & v_{1} \\
v_{2} & v_{1} v_{2}
\end{array}\right) \text { and } \mathbf{A}_{2}=\frac{1}{1+v_{1} v_{2}}\left(\begin{array}{cc}
v_{1} v_{2} & -v_{1} \\
-v_{2} & 1
\end{array}\right)
$$

we can express $\mathbf{A}_{1}$ in the form $\mathbf{T}_{1}^{t} \mathbf{T}_{1}^{\prime}$ and $\mathbf{A}_{2}$ in the form $\mathbf{T}_{2}^{t} \mathbf{T}_{2}^{\prime}$.

Let $\mathbf{T}_{1}=\left(a_{1}, b_{1}\right)$ and $\mathbf{T}_{1}^{\prime}=\left(a_{1}^{\prime}, b_{1}^{\prime}\right)$; then

$$
\mathbf{T}_{1}^{t} \mathbf{T}_{1}^{\prime}=\left(\begin{array}{ll}
a_{1} a_{1}^{\prime} & a_{1} b_{1}^{\prime} \\
b_{1} a_{1}^{\prime} & b_{1} b_{1}^{\prime}
\end{array}\right)
$$

Three equations with four unknowns are obtained

$$
\begin{aligned}
& a_{1} a_{1}^{\prime}=\frac{1}{1+v_{1} v_{2}}, \\
& a_{1} b_{1}^{\prime}=\frac{v_{1}}{1+v_{1} v_{2}},
\end{aligned}
$$




$$
\begin{aligned}
& b_{1} b_{1}^{\prime}=\frac{v_{1} v_{2}}{1+v_{1} v_{2}}, \\
& b_{1} a_{1}^{\prime}=\frac{v_{2}}{1+v_{1} v_{2}} .
\end{aligned}
$$

As in the case of weak coupling, it is possible to fix one of the unknows; for example, in order to respect a reciprocity condition as long as possible, let $a_{1}=1 /\left(\sqrt{1+v_{1} v_{2}}\right)$. Then $1+v_{1} v_{2}>0$, for the parameters of the system verify $\left(\omega_{1}^{2}-\omega_{2}^{2}\right)^{2}+4 k_{1} k_{2}>0$. Then

$$
\begin{aligned}
& a_{1}^{\prime}=\frac{1}{\sqrt{1+v_{1} v_{2}}}, \\
& b_{1}^{\prime}=\frac{v_{1}}{\sqrt{1+v_{1} v_{2}}}, \\
& b_{1}=\frac{v_{2}}{\sqrt{1+v_{1} v_{2}}} .
\end{aligned}
$$

and

$$
\begin{aligned}
& \mathbf{T}_{1}=\frac{1}{\sqrt{1+v_{1} v_{2}}}\left(1, v_{2}\right), \\
& \mathbf{T}_{1}^{\prime}=\frac{1}{\sqrt{1+v_{1} v_{2}}}\left(1, v_{1}\right) .
\end{aligned}
$$

In the same way, for $A_{2}$ :

$$
\begin{aligned}
& \mathbf{T}_{2}=\frac{1}{\sqrt{1+v_{1} v_{2}}}\left(-v_{1}, 1\right), \\
& \mathbf{T}_{2}^{\prime}=\frac{1}{\sqrt{1+v_{1} v_{2}}}\left(-v_{2}, 1\right) .
\end{aligned}
$$

Using equations (34) and (35), the relation (33) then becomes

$$
\left(\begin{array}{c}
X_{n}(\omega) \\
Y_{n}(\omega)
\end{array}\right)=\frac{T_{1}^{t} T_{1}^{\prime}}{m_{1}^{n, k} \Delta I_{1}}\left(\begin{array}{c}
f_{1} \\
f_{2}
\end{array}\right)+\frac{T_{2}^{t} T_{2}^{\prime}}{m_{2}^{n, k} \Delta I_{2}}\left(\begin{array}{c}
f_{1} \\
f_{2}
\end{array}\right)
$$

Thus a modal superposition formula for the two-d.o.f. system with strong coupling has been established.

\section{Remark.}

- $T_{1}$ and $T_{2}$ thus defined are in fact eigenvectors associated with $\lambda_{1}$ and $\lambda_{2}$.

- $T_{1}^{\prime}$ and $T_{2}$ are orthogonal, just as $T_{2}^{\prime}$ and $T_{1}$.

- There is no reciprocity when $k_{1} \neq k_{2}$, but when the matrix $K$ is symmetrical, reciprocity occurs since then $T_{1}=T_{1}^{\prime}$ and $T_{2}=T_{2}^{\prime}$. 


\subsection{TWO RIGID BODIES COLLIDING}

In this section, a mechanical system consisting of two oscillating rigid bodies which can collide during their movement is studied.

It will be assumed that the equilibrium position $x_{1}^{0}$ and $x_{2}^{0}$ of the two solids are such that $x_{2}^{0}-x_{1}^{0}=x_{\max }>0$ (system without preload). The equations of the system in relative displacements are then

$$
\begin{aligned}
& m_{1} \ddot{x}_{1}+c_{1} \dot{x}_{1}+\kappa_{1} x_{1}=g_{1} \cos (\omega t), \\
& m_{2} \ddot{x}_{2}+c_{2} \dot{x}_{2}+\kappa_{2} x_{2}=g_{2} \cos (\omega t), \\
& x_{2}-x_{1} \geqslant x_{\max } .
\end{aligned}
$$

Let $a_{1}=c_{1} / m_{1}, \lambda_{1}=\kappa_{1} / m_{1}, f^{1}=g_{1} / m_{1}$ and similarly $a_{2}=c_{2} / m_{2}, \lambda_{2}=\kappa_{2} / m_{2}, f^{2}=g_{2} / m_{2}$, and assume hereafter that $a_{1}=a_{2}=a$. The system becomes

$$
\begin{aligned}
& \ddot{x}_{1}+a \dot{x}_{1}+\lambda_{1} x_{1}=f^{1} \cos (\omega t), \\
& \dot{x}_{2}+a \dot{x}_{2}+\lambda_{2} x_{2}=f^{2} \cos (\omega t), \\
& x_{2}-x_{1} \geqslant x_{\text {max }} .
\end{aligned}
$$

When $x_{2}(t)-x_{1}(t)=x_{\max }$, an impact occurs at $t$ and the restitution law leads the relative velocity between the two solids to be multiplied by a factor $-e$ at the impact time:

$$
\dot{x}_{2}\left(t^{+}\right)-\dot{x}_{1}\left(t^{+}\right)=-e\left(\dot{x}_{2}\left(t^{-}\right)-\dot{x}_{1}\left(t^{-}\right)\right)
$$

Moreover, the conservation of the momentum provides the second equation to be able to determine post-impact velocities:

$$
m_{2} \dot{x}_{2}\left(t^{+}\right)+m_{1} \dot{x}_{1}\left(t^{+}\right)=m_{2} \dot{x}_{2}\left(t^{-}\right)+m_{1} \dot{x}_{1}\left(t^{-}\right) .
$$

A simple change of co-ordinates can be applied in order to express the system (14) in the form previously studied; let

$$
\begin{aligned}
& x=x_{1}-x_{2}, \\
& y=\frac{m_{1}}{m_{2}} x_{1}+x_{2} .
\end{aligned}
$$
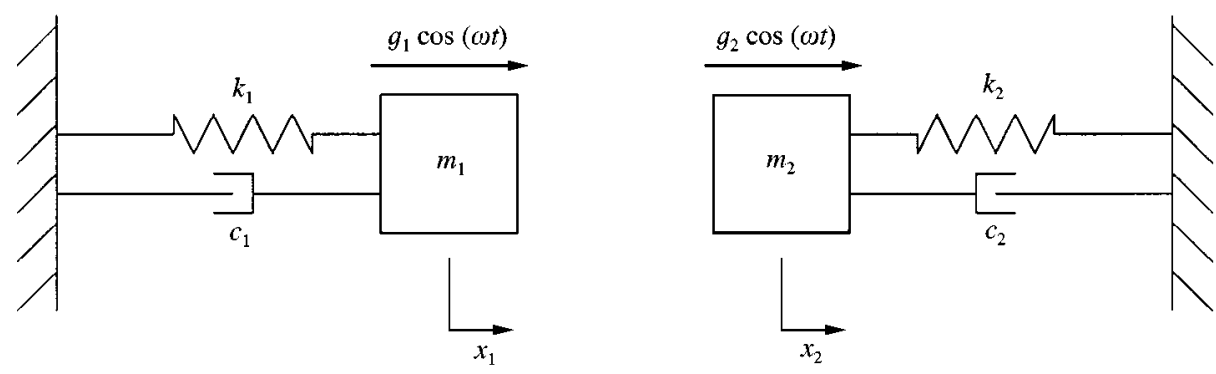

Figure 21. Mechanical model of two rigid oscillating bodies colliding. 
For this new system of variables, the equations become

$$
\begin{aligned}
& \ddot{x}+a \dot{x}+\omega_{1}^{2} x+k_{1} y=f_{1} \cos (\omega t)+\text { "impact", } \\
& \ddot{y}+a \dot{y}+\omega_{2}^{2} x+k_{2} x=f_{2} \cos (\omega t), \\
& x_{2}-x_{1} \geqslant 0,
\end{aligned}
$$

where $\omega_{1}^{2}=\left(m_{1} \lambda_{2}+m_{2} \lambda_{1}\right) /\left(m_{1}+m_{2}\right), \quad \omega_{2}^{2}=\left(m_{1} \lambda_{1}+m_{2} \lambda_{2}\right) /\left(m_{1}+m_{2}\right), \quad k_{1}=\left(m_{2} /\left(m_{1}+\right.\right.$ $\left.\left.m_{2}\right)\right)\left(\lambda_{1}-\lambda_{2}\right), k_{2}=\left(m_{1} /\left(m_{1}+m_{2}\right)\right)\left(\lambda_{1}-\lambda_{2}\right), f_{1}=f^{1}-f^{2}$ and $f_{2}=\left(m_{1} / m_{2}\right) f^{1}+f^{2}$.

Thus the system is of the type (14); the results of the preceding study are then applicable. First of all note that $k_{1}$ and $k_{2}$ are proportional, so that there is always the case of the strong coupling studied in section 3 . As regards the solutions of the system between two impacts, there is no work to do in order to decouple the system, since the initial system is already written in decoupled form. Thus the eigenvalues of the system are always real, and are exactly $\lambda_{1}$ and $\lambda_{2}$.

In this case $v_{1}=1$ and $v_{2}=m_{1} / m_{2}$. According to equations (34) and (35), the modes of the system are given by

$$
\begin{aligned}
& T_{1}=\alpha_{1,2}\left(1, \frac{m_{1}}{m_{2}}\right), \\
& T_{1}^{\prime}=\alpha_{1,2}(1,1), \\
& T_{2}=\alpha_{1,2}(-1,1), \\
& T_{2}^{\prime}=\alpha_{1,2}\left(-\frac{m_{1}}{m_{2}}, 1\right),
\end{aligned}
$$

where $\alpha_{1,2}=1 / \sqrt{1+m_{1} / m_{2}}$.

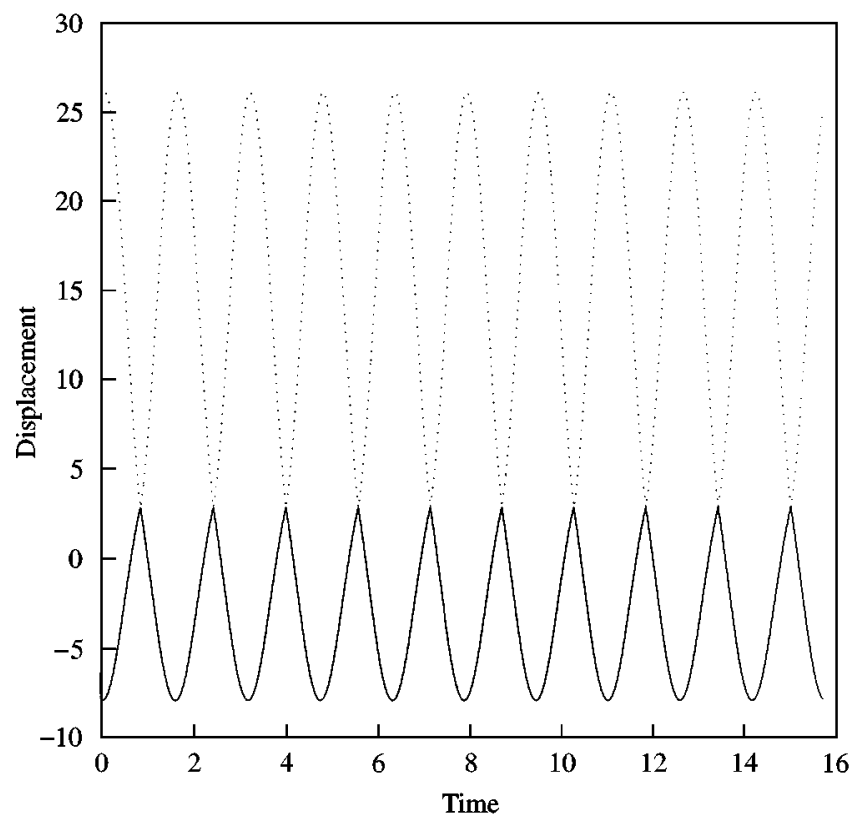

Figure 22. (1,1)-periodic solution for $\omega=4, \quad x_{0}^{1}=-7 \cdot 90482, \quad \dot{x}_{0}^{1}=-2 \cdot 07935, \quad x_{0}^{2}=11 \cdot 20441, \quad$ and $\dot{x}_{0}^{2}=15 \cdot 14127$ : absolute displacement. 

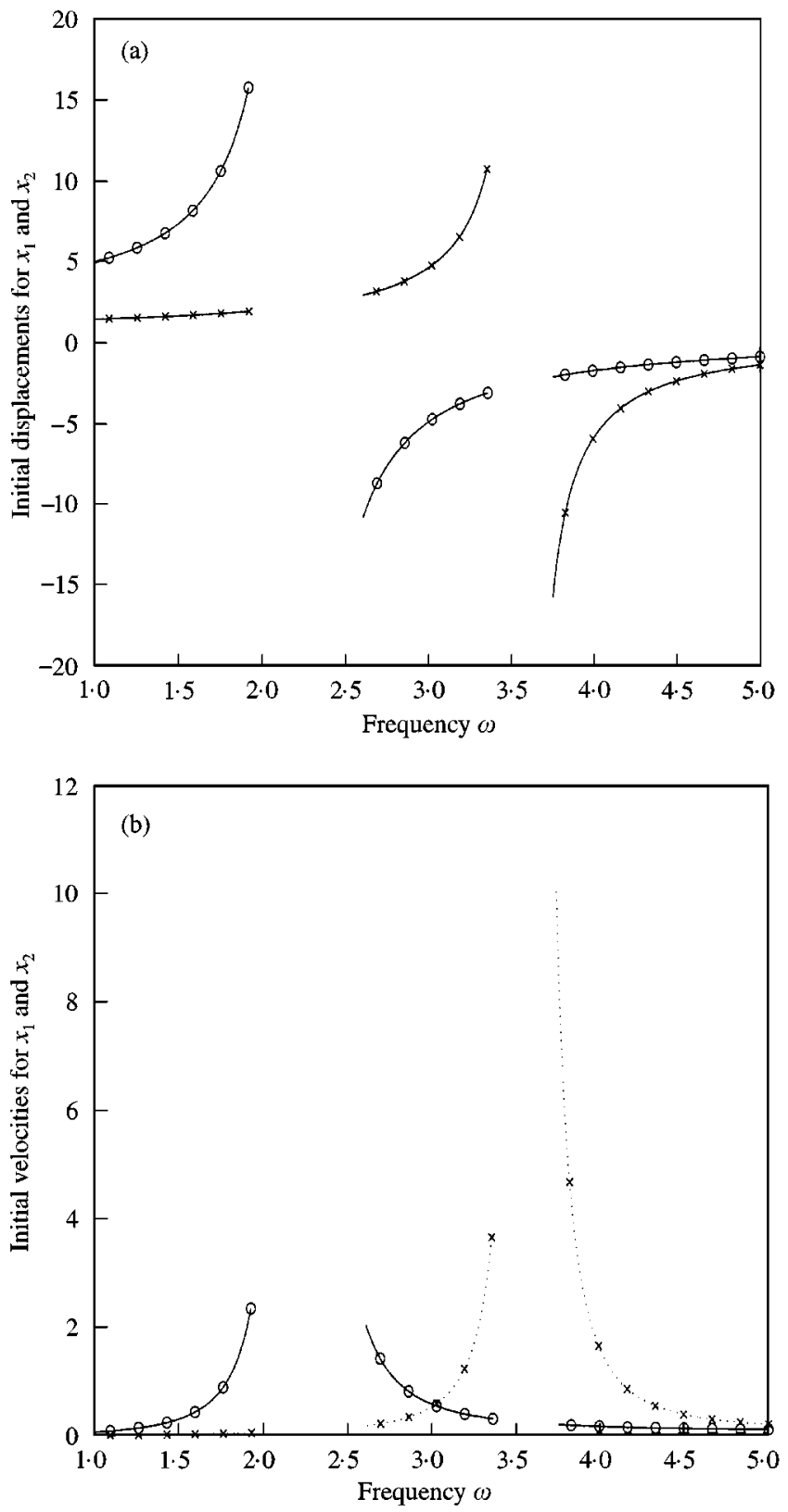

Figure 23. Existence of $(1,0)$-periodic solutions: (a) initial displacements for $x_{1}$ (circle) and $x_{2}$ (x-mark); (b) initial velocities for $x_{1}$ (circle) and $x_{2}$ (x-mark).

Using these modes, the modal superposition formula is as in equation (36), the expression of the modal masses being given by equation (32). It is interesting to note that in general there is no reciprocity, except if $m_{1}=m_{2}$.

Finally, the periodic responses of the system can be dealt with. First of all, according to section 3.2.2, there are $(n, 0)$-periodic solutions because it was assumed that $x_{\max }>0$. In the same way, calculations of section 3.2.2 can search for $(n, 1)$-periodic solutions. Diagrams of existence of periodic solutions versus the frequency of the external excitation are obtained. 

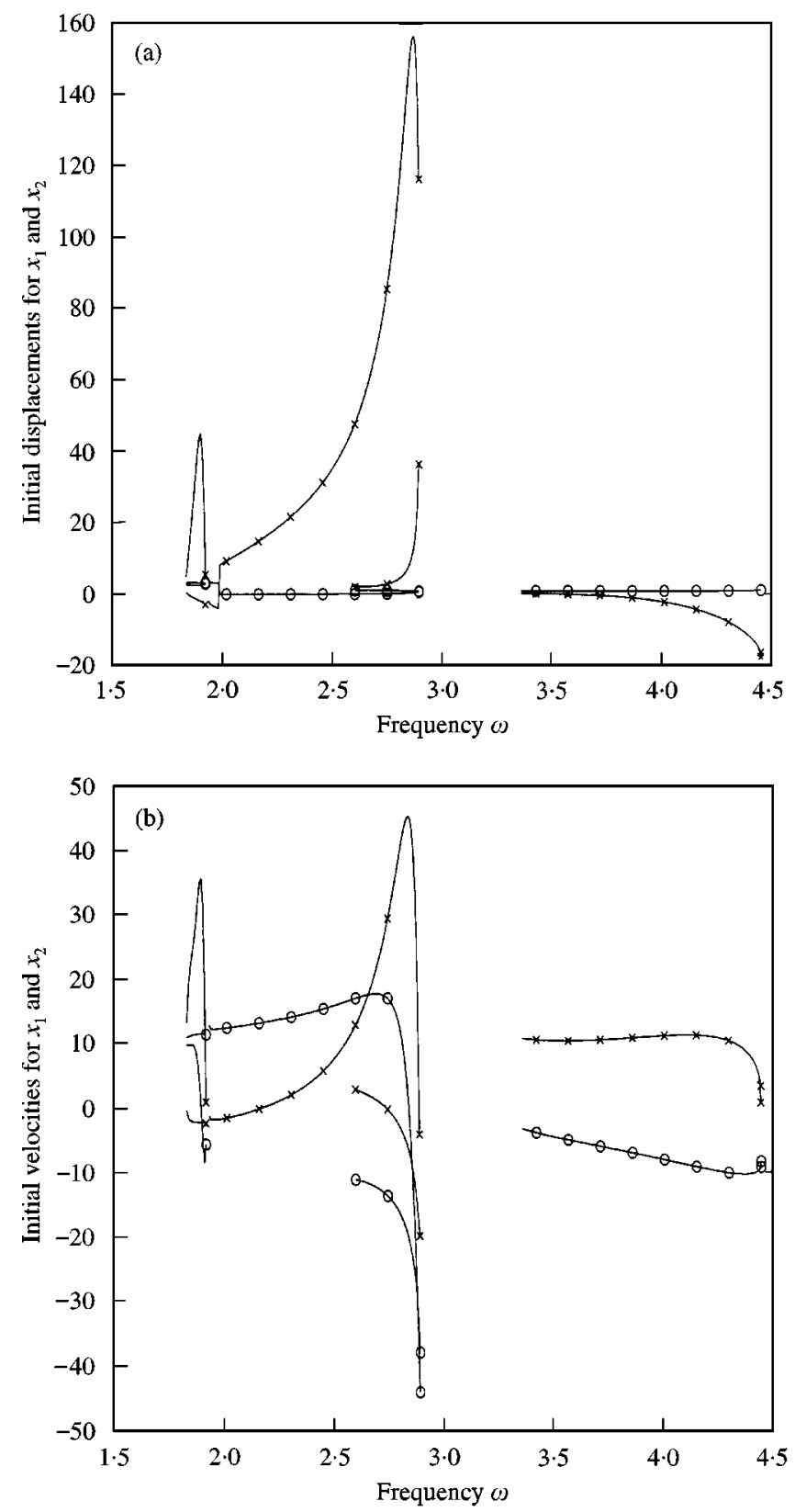

Figure 24. Existence of (1, 1)-periodic solutions: (a) initial displacements for $x_{1}$ (circle) and $x_{2}$ (x-mark); (b) initial velocities for $x_{1}$ (circle) and $x_{2}$ (x-mark).

From these periodic solutions, the modal superposition formula can then be tested, plotting the difference between the spectral amplitude of the system's response and the $n$th harmonic amplitude given by equation (36).

For the first example presented, let $m_{1}=1, m_{2}=0 \cdot 7, \lambda_{1}=5, \lambda_{2}=13, a=0 \cdot 05, e=0 \cdot 9$, $f^{1}=20, f^{2}=18$, and $x_{\max }=14$.

As it was the case for the single-d.o.f. system, note in Figure 25 that the usual resonance of linear systems no longer occurs. Indeed, when the frequency of the external excitation is 

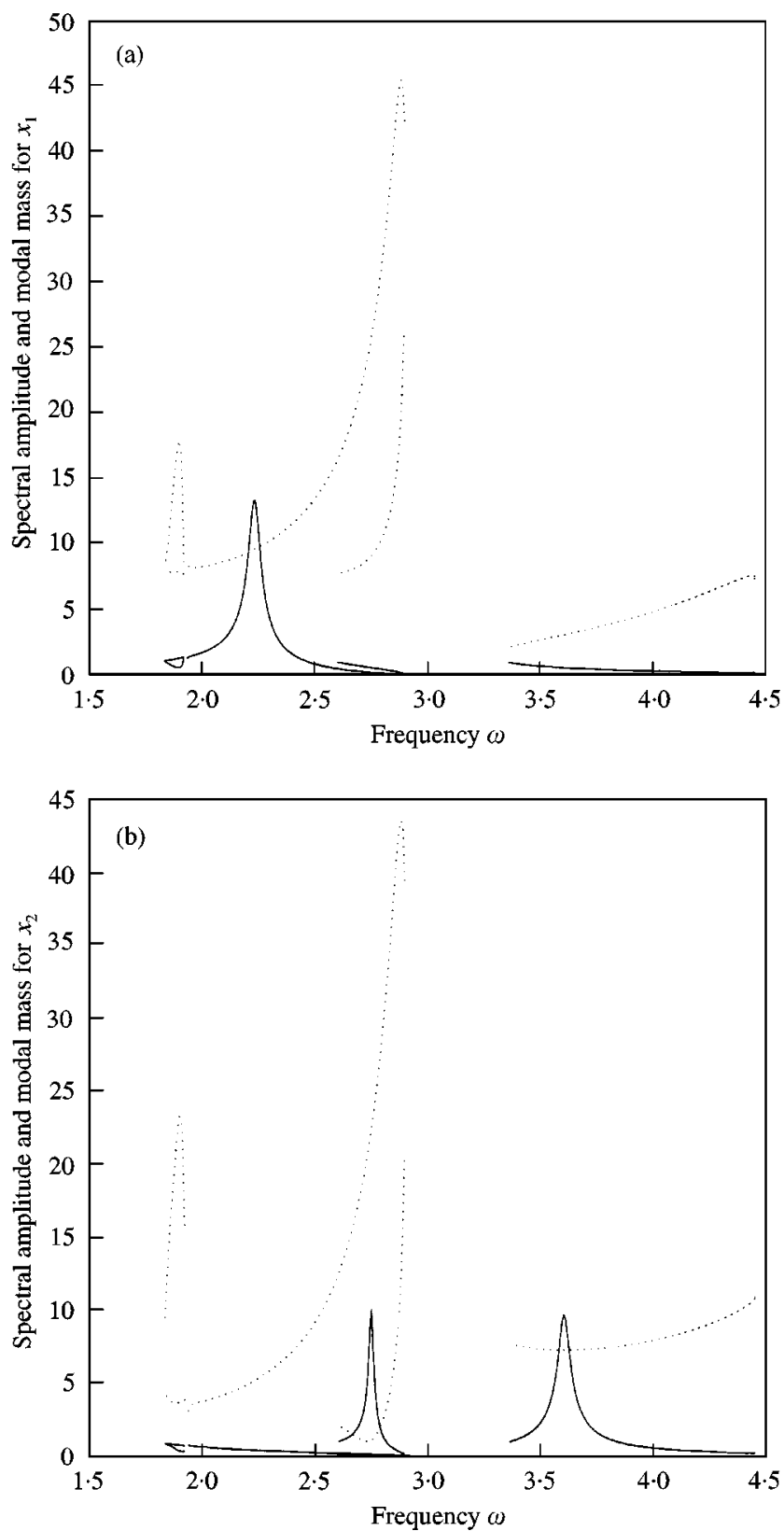

Figure 25. First-harmonic amplitude (dotted curve) and modal mass (solid curve) of the (1, 1)-periodic solutions: (a) first degree of freedom $x_{1}$; (b) second degree of freedom $x_{2}$.

equal to the natural frequency of the system, the modal mass corresponding to the excited mode goes through a local maximum, so that the associated spectral amplitude does not present a particular peak.

Nevertheless, spectral amplitude peaks appear for values of $\omega$ far away from the natural frequencies. The main peak is located in the neighbourhood of $\omega_{2}$ (which is one of the natural frequencies of the system after the change of variables), but it is difficult to show a resonance in $\omega_{2}$, for the peak is appreciably shifted from this frequency. From the modal 

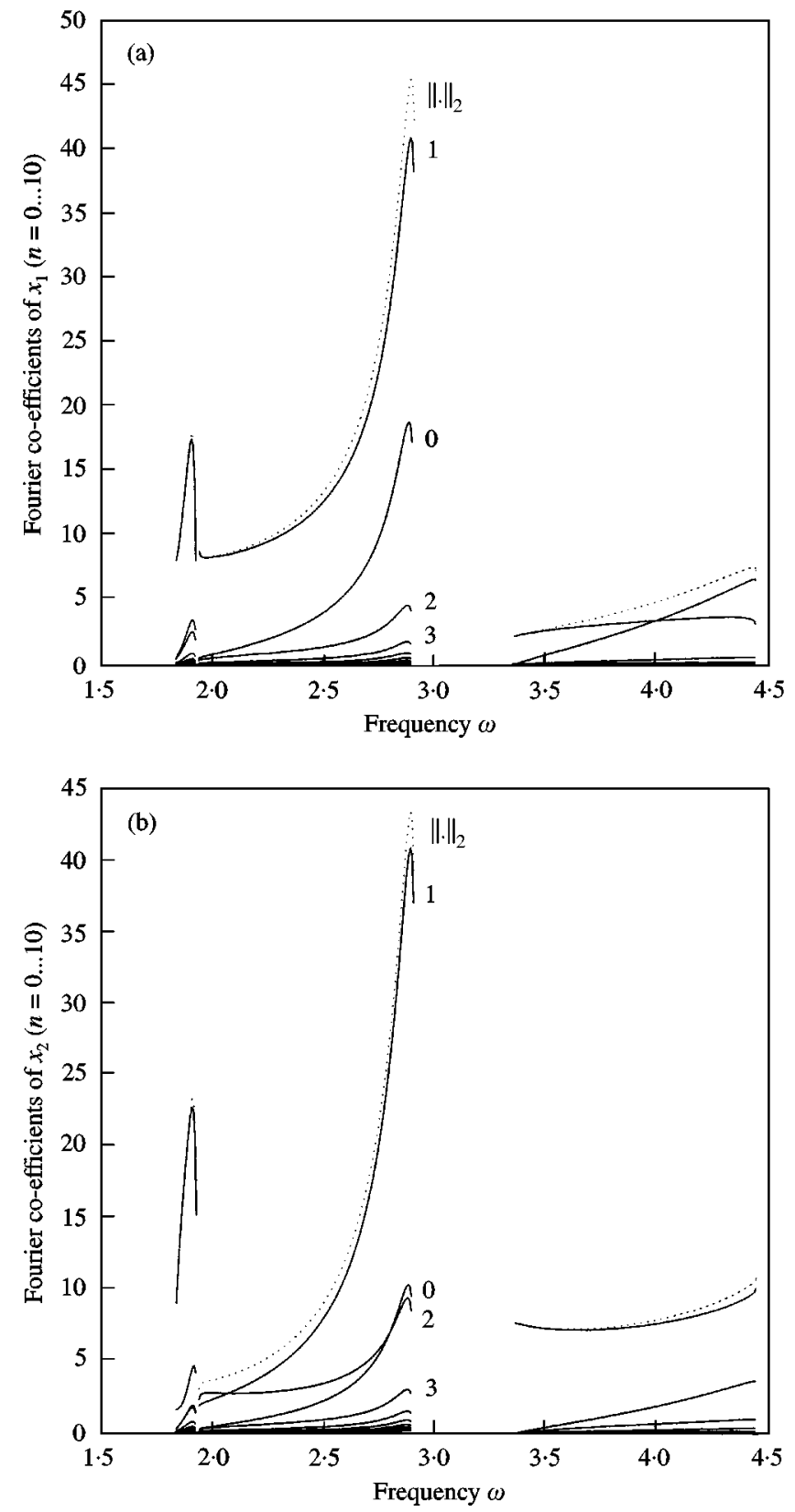

Figure 26. Fourier coefficients of the (1,1)-periodic responses: (a) first degree of freedom $x_{1}$; (b) second degree of freedom $x_{2}$.

point of view, the occurrence of such peaks does not correspond to a classical resonance (where the generalized natural frequency of the system is close to the frequency of external excitation); resonance can be interpreted as the locus of frequency where the generalized modal mass is minimum with respect to $\omega$.

As regards the closeness of the approximation to the spectral amplitude by the $n$th harmonic amplitude, note, as in the single-d.o.f. case, that the first harmonic is not always the leading term in the amplitude of a (1,1)-periodic response (see Figure 26). Thus, the 
TABLE 2

Difference between the whole spectral amplitude and the amplitude of nth harmonic

\begin{tabular}{ccc}
\hline $\begin{array}{c}\text { Degree of } \\
\text { freedom }\end{array}$ & $\begin{array}{c}\text { Difference (in \%) } \\
\text { whole range }\end{array}$ & $\begin{array}{c}\text { Difference (in \%) } \\
\text { neighbourhood of the peak }\end{array}$ \\
\hline$x_{1}$ & $77 \cdot 31$ & $12 \cdot 82$ \\
$x_{2}$ & 82.75 & 4.89 \\
\hline
\end{tabular}
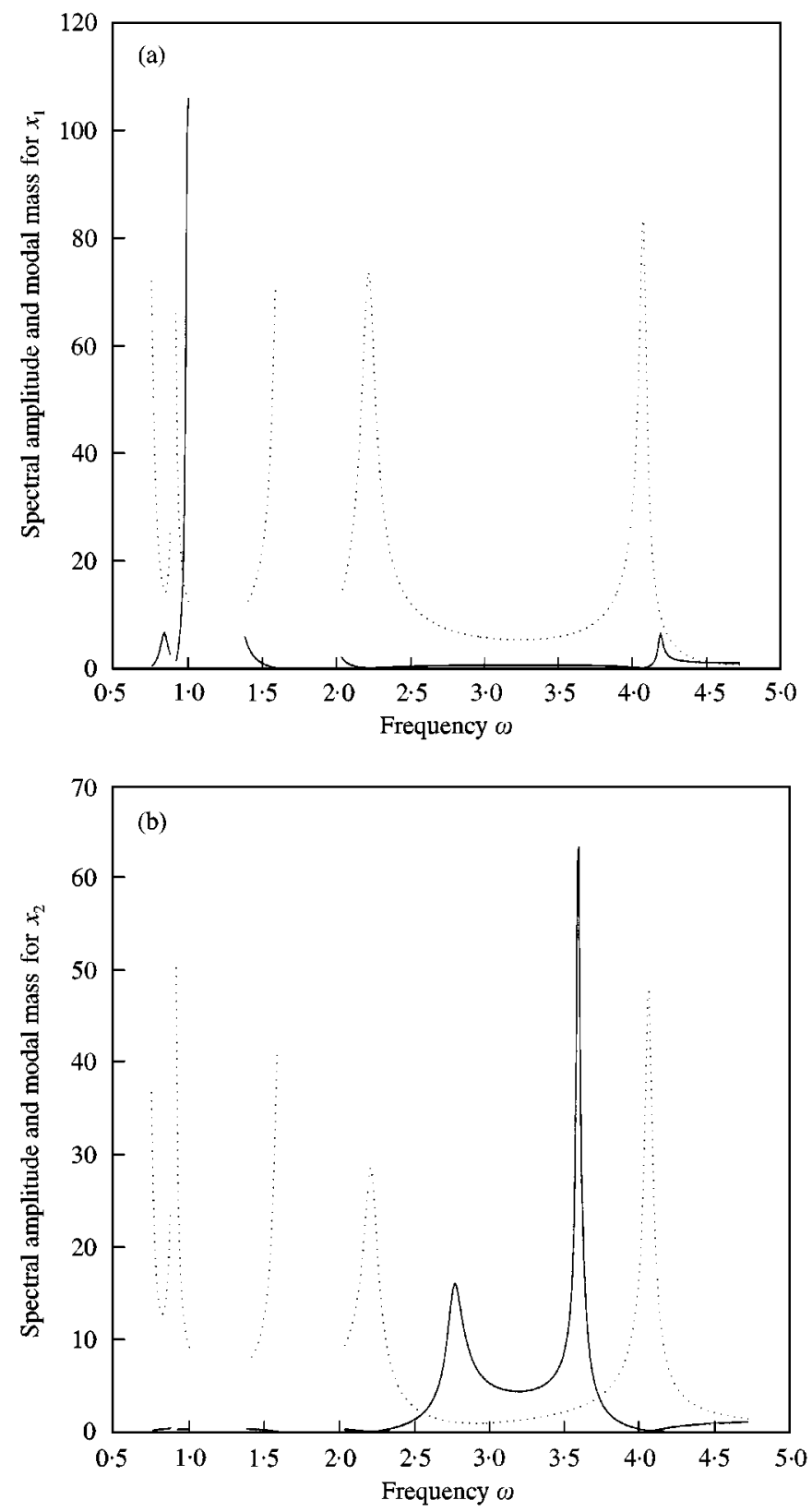

Figure 27. First-harmonic amplitude (dotted curve) and modal mass (solid curve) of the (1,1)-periodic solutions: (a) first degree of freedom $x_{1}$; (b) second degree of freedom $x_{2}$. 

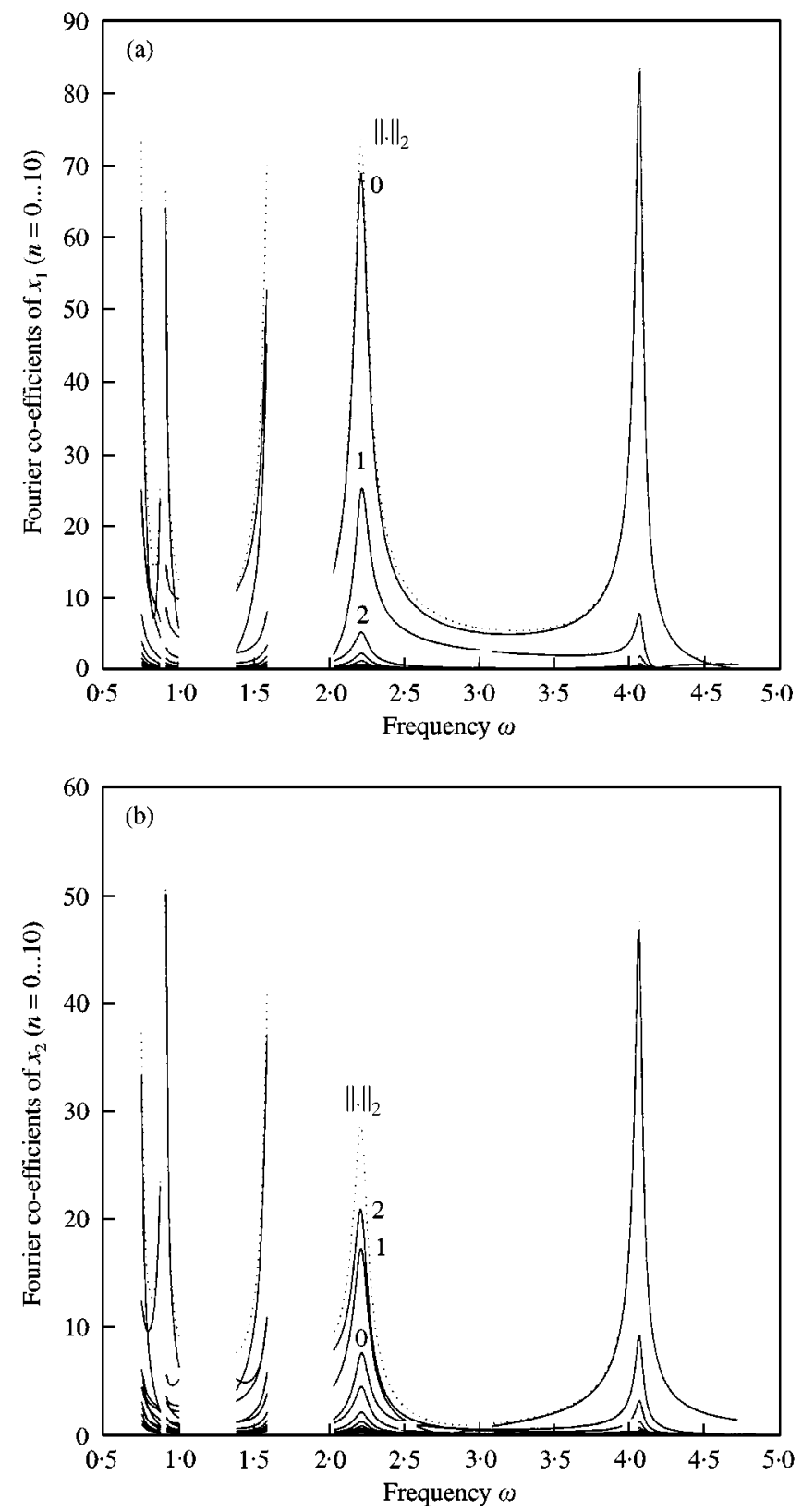

Figure 28. Fourier coefficients of the (1, 1)-periodic responses: (a) first degree of freedom $x_{1}$; (b) second degree of freedom $x_{2}$.

constant coefficient $c_{0}$ overrides all the others for some values of $\omega$. Yet if $\omega$ is considered to be close to the peaks in the spectral response, the first harmonic gives a close approximation to the total amplitude (see Table 2).

For the last example, a set of parameters close to the second example in the single-d.o.f. system is chosen: $m_{1}=1, m_{2}=0 \cdot 7, \lambda_{1}=1, \lambda_{2}=13, a=0 \cdot 02, e=0 \cdot 9, f^{1}=20, f^{2}=18$, and $x_{\max }=1$. Looking at Figures 27 and 28 first note that amplitude peaks appear at the same frequencies for $x_{1}$ and $x_{2}$, which is due to energy transmission between the two bodies 
through impacts. Moreover, the first harmonic amplitude can once again be far lower than the spectral amplitude; most of amplitude peaks in $x_{1}$ come from $\mathscr{A}_{0}$, and for $x_{2}$ the second harmonic is often overriding.

Only the $(1,1)$-periodic responses of the system have been studied. Nevertheless, it must be kept in mind that many other types of periodic solutions are possible, as was seen for the single-d.o.f. system, depending on the time period and the number of impacts per cycle. Theoretically, nothing can prevent a modal superposition formula for any $(n, k)$-periodic response from being written, but in practice such a response is hard to find by analytical means when $k>1$.

\section{CONCLUSION}

The feasibility of building a modal superposition formula for systems with irregular non-linearities of impact type has been investigated, imitating the procedure used in the smooth non-linear case $[49,51]$. The formula has been built for simple single- and two-d.o.f. systems with unilateral constraint and restitution law. The generalized modes and frequencies obtained turn out to be identical to the linear case, the non-linearity of the system being concentrated into the modal masses.

The examples considered show that the formula is valid in the case of a primary resonance for which the spectral amplitude is given by the Fourier coefficient corresponding to the periodicity of the forced solution obtained. Nevertheless, these examples have above all illustrated the limitations to such a formulation. Firstly, the multiplicity of periodic solutions, with different periodicity or number of impacts per cycle, compels several potential formulas to be built, and it is not possible to know a prori which one has to be used. Furthermore, main amplitude peaks may appear away from any a priori clearly identifiable resonance, which may be overidden by some unusual harmonics and consequently cause the modal superposition formula to fail.

Therefore, it is not possible, in a general case, to build a modal superposition formula using only the usual sequence definition of generalized frequencies, definition of generalized modes and then definition of generalized modal masses; the non-linearities of impact type produce a limitation on the formulation of a general formula following the usual procedure.

\section{REFERENCES}

1. T. K. Caughey 1965 Journal of Applied Mechanics September, 583-590. Classical normal modes in damped linear dynamic systems.

2. L. MeIrovitch 1967 Analytical Methods in Vibrations. London: McMillan.

3. L. Jezequel 1985 Ph.D. Thesis, no. 8539. Université Lyon I. Synthèse modale, théorie et extensions.

4. F. Bourquin 1991 Ph.D. Thesis, Université Pierre et Marie Curie. Synthèse modale et analyse numérique des multi-structures élastiques.

5. R. M. Rosenberg and C. P. AtKinson 1959 Journal of Applied Mathematics 26, 377-385. On the natural modes and their stability in non-linear two-degree-of-freedom systems.

6. R. M. Rosenberg 1964 Quaterly of Applied Mathematics XXII, 217-234. On the existence of normal mode vibrations of non-linear systems with two degree-of-freedom.

7. R. M. Rosenberg 1961 Journal of Applied Mechanics 28, 275-283. On normal vibrations of a general class of non linear dual mode systems.

8. R. M. Rosenberg 1962 Journal of Applied Mechanics 29, 7-14. The normal modes of non-linear $n$-degree-of-freedom systems.

9. C. H. PAK and R. M. Rosenberg 1968 Quaterly of Applied Mathematics XXVI, 403-416. On the existence of normal mode vibrations in non-linear systems. 
10. C. H. PAK 1989 Journal of Applied Mechanics 56, 155-161. On the stability behavior of bifurcated normal modes in coupled nonlinear systems.

11. C. H. Cooke and R. A. Struble 1996 Quaterly of Applied Mathematics XXIV, 177-193. On the existence of periodic solutions and normal mode vibrations of non-linear systems.

12. G. V. ANAND 1972 International Journal of Non-linear Mechanics 7, 81-91. Natural modes of a coupled nonlinear system.

13. J. T. StUART 1971 Annual Review of Fluid Mechanics 3, 347-370. Non-linear stability theory.

14. P. A. T. Christopher 1979 Journal of Sound and Vibration 66, 91-97. A modified stroboscopic method for non-linear vibration equations.

15. W. SZEMPLINSKA-STUPNICKA 1969 Archiwun Mechaniki Stosowanej 5, 603-621. On the normal coordinates in an analysis of steady-state forced vibrations of a non-linear multiple-degree-of freedom system.

16. W. SZEmPlinska-STUPNICKA 1983 Journal of Nonlinear Mechanics 18, 149-165. Nonlinear normal modes and the generalised Ritz method in the problems of vibrations of non-linear elastic continuous systems.

17. W. SzemplinskA-STUPNicKA 1990 The behaviour of Non-Linear Vibrating Systems, Vol. 2. Dordrecht: Kluwer.

18. Yu. V. Mikhlin 1996 Nonlinear Dynamics 11, 1-16. Normal vibrations of a general class of conservative oscillators.

19. R. H. RAND 1974 International Journal of Non-Linear Mechanics 9, 363-368. A direct method for non-linear normal modes.

20. A. F. VAKAKIS 1992 Journal of Sound and Vibration 158, 341-361. Nonlinear normal oscillations in a strongly nonlinear discrete system.

21. A. WeINSTEIN 1973 Invertiones Mathematicae 20, 47-57. Normal modes for non-linear hamiltonian systems.

22. A. A. ZEVIN 1984 Journal of Applied Mathematics and Mechanics 48, 451-458. Some conditions for the existence and stability of periodic oscillations in non linear non-autonomous hamiltonian systems.

23. M. R. Hyams and L. A. Month 1984 Journal of Applied Mechanics 51, 399. The origin of stability indeterminacy in a symmetric hamiltonian.

24. T. L. JOHNSON and R. H. RAND 1979 International Journal of Non linear Mechanics 14, 1-12. On the existence and bifurcation of minimal normal modes.

25. L. A. MONTH and R. H. RAND 1977 Journal of Applied Mechanics 44, 782-784. The stability of bifurcating periodic solutions in a two- degree-of-freedom non-linear systems.

26. Y. V. MikHLIN 1995 Journal of Sound and Vibration 182, 577-588. Matching of local expansions in the theory of nonlinear vibrations.

27. A. F. Vakakis, L. I. Manevitch, Y. V. Mikhlin, V. N. Pilipchuk and A. A. Zevin 1996 Normal Modes and Localization in Nonlinear Systems. New York: Wiley.

28. Q. Chen, G. Manson and K. Worden 1997 New Advances in Modal Synthesis of Large Structures (L. Jézéquel, editor), 549-561. Rotterdam: Balkema. Higher-order FRFs for multi-input nonlinear systems: a case study.

29. S. J. GIFFORD and G. R. TOMLINSON 1989 Journal of Sound and Vibration 135, 289-317. Recent developments in the application of functional series to non-linear structures.

30. V. Bargmann 1961 Communications on pure and Applied Mathematics XIV, 187-214. On a Hilbert space of analytic functions and an associated integral transform, Part I.

31. D. Claude 1986 SiAM Journal on Control and Optimization 24, 562-578. Découplage des systèmes non linéaires, séries génératrices non-commutatives et algèbres de Lie.

32. V. I. ARNOLD 1976 Méthodes mathématiques de la mécanique classique. Moscou: Editions de Moscou.

33. F. G. GuSTAVSON 1966 The Astronomical Journal 71, 670. On constructing formal integrals of a Hamiltonian system near an equilibrium point.

34. L. A. Month and R. H. Rand 1980 Journal of Applied Mechanics 47, 645. An application of the Poincare map to the stability of non-linear normal modes.

35. H. Poincare 1987 Les Méthodes Nouvelles de la Mécanique Céleste. Paris: Librairie A. Blanchard.

36. L. HSU 1983 Journal of Sound and Vibrations 89, 169-182. Analysis of critical and post-critical behavior of non linear dynamical systems by the normal form method-Part I. Normalization formulae. 
37. L. HSU 1983 Journal of Sound and Vibrations 89, 183-194. Analysis of critical and post-critical behavior of non linear dynamical systems by the normal form method-Part II. Normalization formulae.

38. H. L. SMith 1986 Journal of Mathematical Analysis and Applications 113, 578-590. Normal forms for periodic systems.

39. L. Hsu 1977 Mechanics Research Communications 4, 219-227. On the analysis of fourth order systems near a bifurcation.

40. H. L. Sмith 1986 SIAM Journal of Mathematical Analysis 17, 1289-1318. Periodic solutions of periodic competitive and cooperative systems.

41. S. W. SHAw 1994 Journal of Nonlinear Science 4, 419-448. An invariant manifold approach to nonlinear normal modes of oscillation.

42. C. PIERRE and S. W. SHAW 1991 Journal of Sound and Vibration 150, 170-173. Non-linear normal modes and invariant manifolds.

43. S. W. SHAW and C. PIERRE 1992 Journal of Sound and Vibration 164, 85-124. Normal modes for non-linear vibratory systems.

44. S. W. SHAW and C. PIERRE 1994 Journal of Sound and Vibration 169, 319-347. Normal modes of vibration for non-linear continuous systems.

45. N. Boivin, C. Pierre and S. W. Shaw 1995 Nonlinear Dynamics 8, 315-346. Non-linear normal modes, invariance, and modal dynamics approximations of non-linear systems.

46. P. J. Holmes and R. H. RAND 1980 International Journal of Non linear Mechanics 15, 387-399. Bifurcation of periodic motions in two weakly coupled Van Der Pol oscillators.

47. P. J. Holmes 1981 Physica D 2, 449-481. Center manifolds, normal forms and bifurcations of vector fields with application to coupling between periodic and steady motions.

48. P. H. Coullet and E. A. Spiegel 1985 SIAM Journal of Applied Mathematics 43, 776-821. Amplitude equation for systems with competing instabilities.

49. L. JezeQuel and C.-H. LAMARQUe 1991 Journal of Sound and Vibrations 149, 429-459. Analysis of nonlinear structural vibrations by normal form theory.

50. L. Jezequel and C.-H. Lamarque 1991 Proceedings of the IXth International Modal Analysis Conference, Florence, Italy, 101-108.

51. C.-H. Lamarque 1992 Ph.D. thesis, no 92-32. Ecole Centrale de Lyon. Contribution à la Modèlisation et à l'Identification des Systèmes Mècaniques Non-Linèaires.

52. C.-H. LAmARQUe and L. JezeQuel Proceedings of the Conference on Experimental and Numerical Methods in Structural Dynamics, 21-25 September 1992, Louvain, Belgium, 937-946. Introduction of nonlinear complex modes by using normal form theory.

53. R. H. RAND, C. PAK and A. F. VAKAKIS 1992 Acta Mechanica 3, 129-145. Bifurcation of nonlinear normal modes in a class of two degree of freedom systems.

54. M. Urabe 1967 Non Linear Autonomous Oscillations, Analytical Theory. New York: Academic Press.

55. G. E. Giachglia 1972 Applied Mathematical Science, Vol. 8. Berlin: Springer. Perturbation methods in nonlinear systems.

56. G. D. Birkhoff 1927 Dynamical Systems, Vol. 9, New York: AMS Coll. Publication.

57. V. N. Bogaevski and A. PovZner 1991 Algebraic Methods in Nonlinear Perturbation Theory. New York: Springer.

58. A. D. BRJuno 1989 Local Methods in Nonlinear Differential Equations. New York: SpringerVerlag.

59. J. CARR 1981 Applications of Center Manifold Theory. New York: Springer-Verlag.

60. F. Verhulst 1996 Nonlinear Differential Equations and Dynamical Systems. Berlin: SpringerVerlag.

61. J. H. GUCKENHEIMER and P. Holmes 1983 Nonlinear Systems and Bifurcations of Vector Fields. New York: Springer-Verlag.

62. J. K. HALE and S. N. CHOW 1982 Methods of Bifurcation. Berlin: Springer-Verlag.

63. J. E. Marsden and M. MCCracken 1976 Applied Mathematical Sciences, Vol. 19. The Hopf bifurcation and its applications. New York: Springer.

64. A. H. NAYFeH 1993 Method of Normal Forms. New York: Wiley.

65. A. H. NAYFEH and D. T. MoOK 1979 Nonlinear Oscillations. New York: Wiley.

66. G. Iooss and D. D. JOSEPH 1985 Elementary Bifurcation and Stability Theory. New York: Springer.

67. N. Sri NAmachChivaYA and Y. K. LiN 1991 International Journal of Non-Linear Mechanics 26, 931-943. Method of stochastic normal forms. 
68. P. BoXler 1991 How to Construct Stochastic Center Manifolds on the level of Vector Fields, in Lyapunov Exponents (L. Arnold, H. Crauel and J.-P. Eckmann, editors), Lecture Notes in Mathematics, Vol. 1486. Berlin: Springer.

69. S.-L. Chen and S. W. SHAW 1996 Nonlinear Dynamics 10, 135-163. Normal modes for piecewise linear vibratory systems.

70. L. ZuO and A. CURNIER 1992 Proceedings of Euromech (L. Jézéquel and C.-H. Lamarque, editors), Vol. 280, 141-148. Rotterdam: Balkema. Dynamics of piecewise linear systems.

71. F. PETERKA and J. VACIK 1992 Journal of Sound and Vibration 154, 95-115. Transition to chaotic motion in mechanical systems with impacts.

72. S. W. SHAW and P. J. Holmes 1983 Journal of Sound and Vibration 90, 129-155. A periodically forced piecewise linear oscillator.

73. S. W. SHAW and R. H. RAND 1989 International Journal of Non-Linear Mechanics 24, 41-56. The transition to chaos in a simple mechanical system.

74. G. S. Whiston 1987 Journal of Sound and Vibration 118, 395-429. Global dynamics of vibro-impacting linear oscillator.

75. A. P. IVANOV 1994 Journal of Sound and Vibration 178, 361-378. Impact oscillations: linear theory of stability and bifurcations.

76. F. Peterka 1996 Proceedings of the Second European Nonlinear Oscillation Conference, Prague, September 9-13. The stability of a symmetric two-degree-of-freedom system with impacts.

77. F. Peterka 1999 Proceedings of the 1999 ASME Design Technical Conferences, September 12-15, Las-Vegas, Nevada, DETC99/VIB-8039. Global analysis of the double impact oscillator dynamics.

78. S. ChATTERJEe and A. K. MALLiK 1996 Journal of Sound and Vibration 191, 539-562. Bifurcations and chaos in autonomous self excited oscillators with impact damping.

79. C. K. Sung and W. S. Yu 1992 Journal of Sound and Vibration 158, 317-329. Dynamics of a harmonically excited impact damper: bifurcations and chaotic motion.

80. A. B. NORDMARK 1991 Journal of Sound and Vibration 145, 279-297. Nonperiodic motion caused by grazing incidence in an impact oscillator.

81. G. S. WhISTON 1992 Journal of Sound and Vibration 152, 427-460. Singularities in vibro-impact dynamics.

82. J. MolenaAr J. G. de Weger and W. v. d. Water 1999 Proceedings of the 1999 ASME Design Technical Conferences, September 12-15, Las-Vegas, Nevada, DETC99/VIB-8036. Near-grazing behaviour of the harmonic oscillator.

83. F. Peterka 1996 Proceedings of the Second European Nonlinear Oscillation Conference, Prague, September 9-13. Dynamics of mechanical systems with impacts and dry friction.

84. F. Peterka 1999 Proceedings of the 1999 ASME Design Technical Conferences, September 12-15, Las-Vegas, Nevada, DETC99/VIB-8350. Analysis of motion of the impact-dry-friction pair of bodies and its application to the investigation of the impact dampers dynamics.

85. F. PFEIFFER 1999 Proceedings of the 1999 ASME Design Technical Conferences, September 12-15, Las-Vegas, Nevada, DETC99/VIB-8346. Impacts with friction. Theory and experiments.

86. K. M. CONE and R. I. ZADOKS 1995 Journal of Sound and Vibration 188, 659-683. A numerical study of an impact oscillator with the addition of dry friction.

87. B. BROGLIATO 1996 Nonsmooth Impact Mechanics. London: Springer.

88. V. I. BABITSKY 1998 Theory of Vibro-Impact Systems and Applications. Berlin: Springer.

89. Y. V. Mikhlin, A. F. VAKaKis and G. SAlenger 1998 Journal of Sound and Vibration 216, 227-250. Direct and inverse problems encountered in vibro-impact oscillations of a discrete system. 